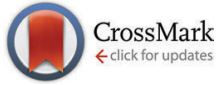

Cite this: Phys. Chem. Chem. Phys., 2017, 19, 9374

Received 7th February 2017 Accepted 7th March 2017

DOI: 10.1039/c7cp00836h

rsc.li/pccp

\title{
Pair natural orbital and canonical coupled cluster reaction enthalpies involving light to heavy alkali and alkaline earth metals: the importance of sub-valence correlation $\dagger$
}

\author{
Yury Minenkov, ${ }^{* a}$ Giovanni Bistoni, ${ }^{b}$ Christoph Riplinger, ${ }^{b}$ Alexander A. Auer, ${ }^{b}$ \\ Frank Neese ${ }^{\star b}$ and Luigi Cavallo*a
}

In this work, we tested canonical and domain based pair natural orbital coupled cluster methods $\operatorname{CCCSD}(T)$ and DLPNO-CCSD(T), respectively) for a set of 32 ligand exchange and association/ dissociation reaction enthalpies involving ionic complexes of $\mathrm{Li}, \mathrm{Be}, \mathrm{Na}, \mathrm{Mg}, \mathrm{Ca}, \mathrm{Sr}, \mathrm{Ba}$ and $\mathrm{Pb}\left({ }^{\prime}\right)$. Two strategies were investigated: in the former, only valence electrons were included in the correlation treatment, giving rise to the computationally very efficient FC (frozen core) approach; in the latter, all non-ECP electrons were included in the correlation treatment, giving rise to the $A E$ (all electron) approach. Apart from reactions involving $\mathrm{Li}$ and $\mathrm{Be}$, the $\mathrm{FC}$ approach resulted in non-homogeneous performance. The FC approach leads to very small errors $\left(<2 \mathrm{kcal} \mathrm{mol}^{-1}\right)$ for some reactions of $\mathrm{Na}, \mathrm{Mg}$, $\mathrm{Ca}, \mathrm{Sr}, \mathrm{Ba}$ and $\mathrm{Pb}$, while for a few reactions of $\mathrm{Ca}$ and $\mathrm{Ba}$ deviations up to $40 \mathrm{kcal} \mathrm{mol}^{-1}$ have been obtained. Large errors are both due to artificial mixing of the core (sub-valence) orbitals of metals and the valence orbitals of oxygen and halogens in the molecular orbitals treated as core, and due to neglecting core-core and core-valence correlation effects. These large errors are reduced to a few $\mathrm{kcal} \mathrm{mol} \mathrm{m}^{-1}$ if the $\mathrm{AE}$ approach is used or the sub-valence orbitals of metals are included in the correlation treatment. On the technical side, the $\operatorname{CCSD}(T)$ and $D L P N O-C C S D(T)$ results differ by a fraction of $\mathrm{kcal} \mathrm{mol}^{-1}$, indicating the latter method as the perfect choice when the CPU efficiency is essential. For completely black-box applications, as requested in catalysis or thermochemical calculations, we recommend the DLPNO-CCSD $(T)$ method with all electrons that are not covered by effective core potentials included in the correlation treatment and correlation-consistent polarized core valence basis sets of cc-pwCVQZ(-PP) quality.

\section{Introduction}

The $\operatorname{CCSD}(\mathrm{T})$ method $^{1}$ is recognized as the gold standard of contemporary electronic structure theory. ${ }^{2,3}$ In its canonical form and with all electrons included in the correlation treatment, $\operatorname{CCSD}(\mathrm{T})$ allows one to achieve chemical accuracy in relative

\footnotetext{
${ }^{a}$ Physical Sciences and Engineering Division, King Abdullah University of Science and Technology, KAUST Catalysis Center, Thuwal 23955-6900, Saudi Arabia. E-mail: Yury.Minenkov@kaust.edu.sa, Luigi.Cavallo@kaust.edu.sa

${ }^{b}$ Department of Molecular Theory and Spectroscopy, Max Planck Institute for Chemical Energy Conversion, Stiftstr. 34-36, D-45470 Mülheim an der Ruhr, Germany. E-mail: Frank.Neese@cec.mpg.de

$\dagger$ Electronic supplementary information (ESI) available: Table S1, cartesian coordinates $(\AA)$, canonical and DLPNO CCSD(T)(AE) and $\operatorname{CCSD}(\mathrm{T})(\mathrm{FC})$ energies with different basis sets, T1 diagnostic values, T2 (largest amplitude values), enthalpic corrections, tabulated enthalpies and errors forming the basis of Charts 1-12, tabulated absolute enthalpies of all 32 reactions and automatically generated auxiliary basis sets needed to run DLPNO-CCSD(T)/cc-pwCV5Z calculations. See DOI: $10.1039 / \mathrm{c} 7 \mathrm{cp} 00836 \mathrm{~h}$
}

electronic energies without any calibration against experimental data as long as the effects of static correlation in the system are sufficiently small. The main shortcoming of this protocol is that it can only be applied to relatively small systems, since it scales as $\mathrm{N}^{7}$ with $\mathrm{N}$ being a measure of system size.

There are two main approximations to reduce the prohibitive computational cost and make routine $\operatorname{CCSD}(\mathrm{T})$ calculations feasible for systems of practical interest. The first approximation takes advantage of resolution of identity (RI) techniques and new algorithms for the two-electron integral transformation ${ }^{4-7}$ together with the careful exploitation of localized molecular orbitals ${ }^{8-11}$ to optimize the selection of the most relevant excitations. ${ }^{12-15}$ In particular, the so-called domain based pair natural orbitals DLPNO-CCSD(T) method ${ }^{9-10,16}$ is especially promising for large-scale computational chemistry applications given its accuracy, efficiency, robustness and ready availability. ${ }^{17}$ The second approximation is the so called "frozen core" (FC) approach, when only valence orbitals are included in the correlation treatment, thus reducing 
the computational cost dramatically. Usually, this approach is physically sound since dynamic correlation in the core electrons does not affect relative energies significantly. Taken together, the DLPNO-CCSD(T) in conjunction with the FC approach enables coupled cluster calculations on molecules containing hundreds of heavy atoms.

The accuracy of the first approximation has been tested in several studies, ${ }^{7,15,18-20}$ and the main conclusion is that with tight pair natural orbital (PNO) settings as part of the DLPNO-CCSD(T) algorithm, the accuracy of the canonical $\operatorname{CCSD}(\mathrm{T})$ approximation can be approached to within a fraction of a $\mathrm{kcal} \mathrm{mol}^{-1}$. By contrast, systematic calculations ${ }^{21-27}$ of the heats of formation via $\operatorname{CCSD}(\mathrm{T})$-based composite schemes have revealed that in several cases the FC approach results in deviations up to 30-40 $\mathrm{kcal} \mathrm{mol}^{-1}$ with respect to the experimental data. Thus, Radom and co-workers $^{22,23}$ have found that $\mathrm{G} 2^{28,29} \mathrm{QCISD}(\mathrm{T})$ and $\operatorname{CCSD}(\mathrm{T})$ methods fail to reproduce the experimental heats of formation of several highly polar oxides and hydrides of alkali ( $\mathrm{Li}-\mathrm{K}$ ) and alkaline earth (Be-Ca) metals, with errors greater than $25 \mathrm{kcal} \mathrm{mol}^{-1}$. The authors found that expanding the correlation space on the metals from the default "frozen core" approach, which includes only valence electrons, to a "reduced frozen core" approach, thus including also sub-valence electrons, drastically improves the situation. For $\mathrm{K}$ and $\mathrm{Ca}$ compounds, inclusion of the sub-valence $3 \mathrm{~s}$ and $3 \mathrm{p}$ orbitals turned out to be essential. The most accurate results have been obtained with the correlation consistent core-valence basis sets (cc-pwCVnZ) of Peterson and co-workers. ${ }^{30,31}$ Moreover, the poor performance of the standard frozen core approach for complexes involving alkaline and alkalineearth metals has been documented earlier by Martin et $a .^{32}$ Similarly, Bauschlicher and co-workers have demonstrated that the standard frozen core approximation leads to large errors in G2 atomization energies of Ga oxides and fluorides. ${ }^{21}$ Such errors appear due to inversion of the Ga $3 \mathrm{~d}$ core orbital and the $2 \mathrm{~s}$ valence orbitals of $\mathrm{O}$ or $\mathrm{F}$. Adding the Ga $3 \mathrm{~d}$ orbital to the correlation treatment significantly improved the situation. Finally, Dixon and co-workers have shown that the Hf $4 \mathrm{f}$ core orbitals can mix with the valence orbitals of oxygen and fluorine, leading to errors in the heat of formation of the corresponding binary compounds. ${ }^{33}$ This is especially disappointing as the $\mathrm{Hf}$ ff orbitals have been included in the widely used fully relativistic Stuttgart-Dresden effective core potential (ECP), ${ }^{34}$ which is used with the corresponding corevalence cc-pwCVnZ-PP basis set of Peterson and co-workers. ${ }^{34}$ The only way to include $4 \mathrm{f} \mathrm{Hf}$ electrons in the correlation is through the application of the scalar-relativistic all electron basis set developed by Peterson and co-workers, ${ }^{34}$ which results in very large computational time even with the cc-pwCVTZ-DK basis set. To overcome these issues, an automatic procedure has been proposed by Austin et al. ${ }^{25}$ In this approach, an overlap criterion is introduced that connects the detection of core orbitals in the molecule, which might be difficult sometimes, to that in the isolated atoms, which is well defined. The procedure has been shown to remove errors arising from the inconsistent treatment of the core orbitals in gallium fluorides. ${ }^{21}$ In addition, the scheme was shown to be able to identify strong mixing between core and valence orbitals, which also might result in large errors, and can only be ameliorated by extension of the correlation space. Thus, there is evidence to suspect that in a significant number of cases, the frozen core approach can deteriorate the results. That is why practically all contemporary composite schemes, ${ }^{35-38}$ in particular the correlation consistent composite approach (ccCA) of Wilson and co-workers, ${ }^{39}$ the Feller-PetersonDixon (FPD) approach ${ }^{27,35-37}$ and the Weizmann-n theories, ${ }^{40}$ involve a step in which all sub-valence electrons are included in the correlation treatment, although sometimes with only triple- $\zeta$ quality basis sets due to reasons of affordability.

It has to be noted that despite documented failures, the standard FC approach is still default in many quantum chemistry computer codes such as ORCA, ${ }^{17}$ Priroda $^{41}$ and Gaussian. ${ }^{42}$ Truhlar and co-workers have shown that the frozen core approach is not responsible for the failures of $\operatorname{CCSD}(\mathrm{T})$ in reproducing the experimental bond dissociation energies of small molecules of 1st row transition metals. ${ }^{43}$ Subsequent studies indicated that the documented failure can be related to insufficiently large basis sets. ${ }^{4,45}$ Indeed, we have recently shown that many reaction enthalpies involving transition metals were not affected by the frozen core approach ${ }^{18,46}$ We do not exclude that in many cases the surprisingly good performance of the FC approach might result from the lucky cancellation of errors from neglecting core and core-valence correlation in both reactants and products. However, few reactions turned out to be very sensitive to the frozen core settings, leading to errors of $30-40 \mathrm{kcal} \mathrm{mol}^{-1}$ when the default FC settings were used, for example reactions of $\mathrm{ZrF}_{4}$ with $\mathrm{Cl}_{2}, \mathrm{Br}_{2}$ or $\mathrm{I}_{2}$.

One way to address these issues and to preserve the performance of the $\operatorname{CCSD}(\mathrm{T})$ method is to carefully examine the molecular orbitals in the system and manually set up the orbitals included in the correlation treatment. While this strategy can lead to reliable results at only somewhat higher computational costs, the application of $\operatorname{CCSD}(\mathrm{T})$ as a black-box method is lost. If the scope of the work is examining and comparing several systems, for example when building reaction pathways, the calculations become impractical again.

An alternative to manual orbital selection is inclusion of all the electrons involved in the SCF procedure also in the post-SCF correlation treatment. Despite the increased computational costs compared to manual orbital selection, it preserves black-box application of the $\operatorname{CCSD}(\mathrm{T})$ method to routine calculations.

In this work we have carefully selected reaction enthalpies for which the effects from core correlation could be large for various reasons, including (a) only a few valence electrons on the metal, (b) high-lying 'core' orbitals or (c) high ionicity of the bonds (e.g. see the ligand exchange reactions of $\mathrm{ZrF}_{4}$ studied previously $\left.{ }^{46}\right)$. These are 32 inorganic ligand exchange and association reactions involving halides, hydrides and oxides of non-transition metals ( $\mathrm{Li}, \mathrm{Be}, \mathrm{Na}, \mathrm{Mg}, \mathrm{Ca}, \mathrm{Sr}, \mathrm{Ba}$ and $\mathrm{Pb}$ ) for which accurate experimental gas phase enthalpies are available. The effect of the FC approximation has been inspected for all the reactions by comparisons with all electron results and experimental values. Alongside with canonical $\operatorname{CCSD}(\mathrm{T})$, the quality of the DLPNO-CCSD(T) approximation has been investigated for its ability to recover the effects of core correlation. 


\section{Computational details}

\subsection{Geometry optimization}

All geometry optimizations were performed with the GGA PBE ${ }^{47,48}$ functional and the all electron triple- $\zeta$-quality basis sets $\lambda_{2}{ }^{49}$ as implemented in the Priroda 13 suite of programs. ${ }^{41}$ Scalar relativistic effects were taken into account via the Dyall Hamiltonian. ${ }^{50}$ The default adaptively generated Priroda grid, corresponding to an accuracy of the exchange-correlation energy per atom $\left(1 \times 10^{-8}\right.$ Hartree), was decreased by a factor of 100 for more accurate evaluation of the exchange-correlation energy. Default values were used for the self-consistent-field (SCF) convergence and the maximum gradient for geometry optimization criterion $\left(1 \times 10^{-4} \mathrm{au}\right)$, whereas the maximum displacement geometry convergence criterion was decreased to 0.0018 au. Geometries were characterized as true energy minima by the eigenvalues of the analytically calculated Hessian matrix. Translational, rotational, and vibrational partition functions for thermal corrections to arrive at total enthalpies were computed within the ideal-gas, rigid-rotor, and harmonic oscillator approximations. The temperature used in the calculations of thermochemical corrections was set to $298.15 \mathrm{~K}$ in all the cases.

\subsection{Single-point energy evaluations}

All single-point energy evaluations were performed with canonical CCSD(T) as well as DLPNO-CCSD(T) methods as implemented in the ORCA suite of programs. ${ }^{17}$ The tight PNO settings (TCutPairs = $10^{-5}$, TCutPNO $=1 \times 10^{-7}$, TCutMKN $=10^{-3}$ ) were used to reduce any numerical noise in the calculations. The default SCF convergence criterion NormalSCF (energy change $1 \times 10^{-6}$ au) was replaced with tighter VeryTightSCF (energy change $1 \times 10^{-9}$ au) to achieve better converged wave-functions. In some cases, this led to fewer subsequent CCSD iterations.

For all-electron calculations, some special precautions need to be taken in order to achieve high accuracy in DLPNO-CCSD(T) calculations relative to calculations involving only valence electrons. These led to some minor modifications in the procedure that will be detailed elsewhere ${ }^{51}$ and will be included in the upcoming ORCA 4.0 release. In practice, for the electron pairs containing core electrons, a lower TCutPNO threshold must be used in order to include a sufficient number of virtual orbitals in the correlation treatment. In the present work, a TCutPNO value of $10^{-9}$ has been used for the electron pairs containing at least one core electron.

To explore the impact of tighter PNO settings on the computational efficiency of the DLPNO-CCSD(T) approximation compared to canonical $\operatorname{CCSD}(\mathrm{T})$, the wall execution times for the $\operatorname{CCSD}(\mathrm{T})(\mathrm{AE}), \operatorname{CCSD}(\mathrm{T})(\mathrm{FC})$, DLPNO-CCSD(T)(AE) and DLPNO-CCSD(T)(FC) protocols, using the $\mathrm{PbI}_{2}$ molecule (ECPs on $\mathrm{Pb}$ and I with cc-pwCVQZ-PP basis sets) as the test case, were measured. These calculations were carried out using a single computer node under Linux OS with the following hardware details: HP DL585 G7 Quad sockets AMD Opteron 6376, 16 cores/socket $2.3 \mathrm{GHz}, 1.5$ Terabyte of random access memory (RAM). In all calculations we used 32 physical cores with $20 \mathrm{~Gb}$ of RAM per core, resulting in total used RAM of $640 \mathrm{~Gb}$. The rest of RAM was used to emulate the hard drive (/dev/shm/) to speed up all I/O operations. All the calculations started exactly from the same HF wave function and no SCF iteration was performed (noiter keyword). As expected, the DLPNO-CCSD(T)(FC) calculation turned out to be the fastest and took $2825 \mathrm{~s}$. We set this as the reference time. The CCSD(T)(FC) calculation took exactly two times longer, which results in a ratio in the wall execution times of 2.0. The DLPNO-CCSD(T)(AE) calculation is 3.8 times slower, indicating that inclusion of all the electrons in the correlation comes at a certain price. Finally, the $\operatorname{CCSD}(\mathrm{T})(\mathrm{AE})$ calculation is 8.4 times slower. Summing up these results indicates that the DLPNO scheme with tight PNO settings is two times faster compared to the canonical CCSD(T) protocol. It should be pointed out that for small systems with only three atoms the DLPNO approximation is not expected to significantly speed up the $\operatorname{CCSD}(\mathrm{T})$ calculations. Due to the linear scaling of DLPNO$\operatorname{CCSD}(\mathrm{T})$ and the $\mathrm{N}^{7}$ scaling of canonical $\operatorname{CCSD}(\mathrm{T})$, however, the speedup of the DLPNO approximation is becoming significant for systems with more than five to ten heavy atoms. Already for medium sized systems with more than 30 to 40 atoms, canonical $\operatorname{CCSD}(\mathrm{T})$ calculations are not feasible at all, whereas DLPNO-CCSD(T) calculations are carried out in a routine fashion on large systems with several hundreds of atoms. ${ }^{10,16}$

2.2.1 Correlation consistent basis sets. The following triple- $\zeta$, quadruple- $\zeta$ and $5-\zeta$ (TZ, QZ and $5 \mathrm{Z}$ ) correlation consistent basis sets were used in the present work (see Section 3.1 for a benchmark study of the performance of different families of basis sets): Calculations involving the $5 \mathrm{Z}$ basis set were only performed at the DLPNO-CCSD(T) level due to hardware/CPU time limitations. Hydrogen was described with the cc-pVnZ basis sets of Dunning. ${ }^{52}$ Oxygen, fluorine, chlorine, lithium, beryllium, sodium, and magnesium atoms were described with the all electron correlation consistent polarized core valence cc-pwCVnZ basis sets of Peterson and co-workers. ${ }^{30,31}$ The calcium atom was described with the all electron correlation consistent polarized core valence cc-pwCVnZ basis set of Koput and Peterson. ${ }^{53}$ Strontium and barium were described with the pseudopotential-based correlation consistent polarized core valence cc-pwCVnZ-PP basis sets of Peterson and co-workers. ${ }^{54}$ The twenty eight electrons of $\mathrm{Sr}$ and 46 electrons of Ba were described with Stuttgart-type fully relativistic effective core potentials..$^{55}$ Bromine and iodine were described with pseudopotential-based correlation consistent polarized core valence cc-pwCVnZ-PP basis sets of Peterson and co-workers. ${ }^{56}$ The 10 electrons of $\mathrm{Br}$ and 28 electrons of I were described with fully relativistic Stuttgart-type pseudopotentials. ${ }^{57,58}$ Finally, $\mathrm{Pb}$ was described with the pseudopotential-based correlation consistent polarized core valence cc-pwCVnZ-PP basis sets of Peterson et al. ${ }^{56}$ The 60 electrons of $\mathrm{Pb}$ were described with the fully relativistic Stuttgart-type potential of Metz and co-workers. ${ }^{59}$ These basis sets were used with both canonical and DLPNO-CCSD(T) implementations. The correlation fitting basis sets def2-qzvpp/ C developed by Hättig, ${ }^{60,61}$ necessary for the resolution of identity approximation as part of the DLPNO scheme, were used together with the TZ and QZ basis sets. All def2/C basis sets were downloaded from the official web page of the Turbomole group. ${ }^{62}$ For the $5 \mathrm{Z}$ calculations the AutoAux ${ }^{63}$ automatic auxiliary basis set construction option of the ORCA 4.0 package 
was used which differs considerably from the automatically generated auxiliary basis sets used in earlier ORCA versions (see ESI $\dagger$ for the basis sets). Both frozen core and all (non-ECP) electron approximations were used.

2.2.2 Complete basis set extrapolation. To eliminate the effects of basis set incompleteness, we used the following extrapolation schemes for HF and correlation energies of individual species suggested by Helgaker. ${ }^{64-66}$ For two adjacent TZ/QZ level basis sets and QZ/5Z basis sets (only for DLPNO-CCSD(T)):

$$
\begin{gathered}
E_{\mathrm{HF}}^{X}=E_{\mathrm{HF}}^{\infty}+\alpha \mathrm{e}^{-1.63 X} \\
E_{\text {corl }}^{X}=E_{\text {corl }}^{\infty}+\beta X^{-3}
\end{gathered}
$$

where $X=3$ and 4 for TZ and QZ basis sets and $X=4$ and $X=5$ for QZ and $5 \mathrm{Z}$ basis sets, respectively; $E_{\mathrm{HF}}^{\infty} / E_{\mathrm{corl}}^{\infty}$ are the $\mathrm{HF}$ and correlation energies at the CBS limit; $\alpha / \beta$ are parameters to be obtained from the two-equation system.

The total enthalpy at the CBS limit for each reaction $(\mathrm{A}+\mathrm{B} \rightarrow \mathrm{C}+\mathrm{D})$ was evaluated via the following equation:

$$
\begin{aligned}
\Delta H_{\mathrm{CCSD}(\mathrm{T})}^{0}= & E_{\mathrm{HF}}^{\infty}(\mathrm{C})+E_{\mathrm{corl}}^{\infty}(\mathrm{C})+H_{\mathrm{corr}}^{\mathrm{PBE}}(\mathrm{C})+E_{\mathrm{HF}}^{\infty}(\mathrm{D})+E_{\mathrm{corl}}^{\infty}(\mathrm{D}) \\
& +H_{\mathrm{corr}}^{\mathrm{PBE}}(\mathrm{D})-\left(E_{\mathrm{HF}}^{\infty}(\mathrm{A})+E_{\mathrm{corl}}^{\infty}(\mathrm{A})+H_{\mathrm{corr}}^{\mathrm{PBE}}(\mathrm{A})+E_{\mathrm{HF}}^{\infty}(\mathrm{B})\right. \\
& \left.+E_{\mathrm{corl}}^{\infty}(\mathrm{B})+H_{\mathrm{corr}}^{\mathrm{PBE}}(\mathrm{B})\right) .
\end{aligned}
$$

where $H_{\text {corr }}^{\mathrm{PBE}}$ is the correction to the electronic energy to arrive at the enthalpy obtained with the standard harmonic oscillator/ rigid rotor/ideal gas approximation (see Section 2.1 for details).

\subsection{The benchmark set}

To accurately benchmark electronic structure theory methods, high quality gas phase experimental data are requested. Gas phase formation enthalpies of many compounds, among which are 1 and 2 group metal complexes as well as $\mathrm{Pb}$ (II), are measured and tabulated, ${ }^{67,68}$ and are used as benchmarks. ${ }^{69-75}$ In this work, however, we do not try to reproduce the absolute formation enthalpies for a few reasons. First, direct calculation of heats of formation via atomization schemes cannot be done via the DLPNO-CCSD(T) method, which is only available for singlet ground states so far, while many atoms have open-shell ground states. Second, direct calculation of formation enthalpies via atomization schemes often leads to larger errors compared to the reaction-based schemes since electron correlation energies in atoms and molecules are very different and no error-compensation can be expected. $^{2}$ To overcome these difficulties, we created reaction datasets from experimentally obtained individual formation enthalpies and validated the electronic structure methods against these datasets (Table 1).

The enthalpies used to build the reaction datasets have to be accurate, since the overall uncertainty for the reaction enthalpy will be the algebraic sum of the uncertainties of reactants and products. Due to these circumstances, in this work we only used the experimental formation enthalpies of 1 and 2 group metal complexes for which few experimental measurements exist and agree with each other. Then, the resulting formation enthalpies were taken as average of all available experimental measurements. Only the enthalpies with an error bar smaller than $4.6 \mathrm{kcal} \mathrm{mol}^{-1}$ were taken. Moreover, the experimental enthalpies for which a large $\left(>5 \mathrm{kcal} \mathrm{mol}^{-1}\right)$ disagreement with $\operatorname{CCSD}(\mathrm{T})$ calculations in the literature were detected have been excluded from analysis. For example, the experimental formation enthalpy for $\mathrm{PbF}_{2}$ is ca. $-104.2\left(-104.0 \pm 2.0 ;^{68}\right.$ $\left.-104.5 \pm 2.0 ;^{76,77}-102.4 \pm 2.2 ;^{78}-106.0^{79}\right)$ while theoretical $\operatorname{CCSD}(\mathrm{T})$ calculations of Dixon et al. ${ }^{80}$ predicted this enthalpy to be $-110.7 \mathrm{kcal} \mathrm{mol}^{-1}$. We combined these enthalpies to obtain the overall uncertainties in reaction enthalpies $\left( \pm 5 \mathrm{kcal} \mathrm{mol}^{-1}\right)$. In order to evaluate the error bars for reaction enthalpies we proceeded as follows. For every reaction $\mathrm{A}+\mathrm{B} \rightarrow \mathrm{C}+\mathrm{D}$, first the formation enthalpies of $\mathrm{C}$ and $\mathrm{D}$ were taken at their overestimated values, while for $\mathrm{A}$ and $\mathrm{B}$ the formation enthalpies were taken at their underestimated values. For example, if the formation enthalpy of $\mathrm{C}$ is $5.0 \pm 2.0 \mathrm{kcal} \mathrm{mol}^{-1}$, we took it as $7.0 \mathrm{kcal} \mathrm{mol}^{-1}$ and if the formation enthalpy of $\mathrm{A}$ is $3.0 \pm 2.0$ we took it as $1.0 \mathrm{kcal} \mathrm{mol}^{-1}$. Then, we did the inverse, namely the formation enthalpies of $\mathrm{C}$ and $\mathrm{D}$ were taken at their underestimated values, while for $\mathrm{A}$ and $\mathrm{B}$ the formation enthalpies were taken at their overestimated values. The reaction enthalpies were taken at the average of these estimates, and uncertainties corresponded to the lowest and highest reaction enthalpies. This methodology allows us to consider in all reactions the most pessimistic error bars for both highest and lowest uncertainties. The reaction enthalpies are tabulated in the ESI, $\dagger$ and are also available at the url: https:// sites.google.com/site/theochemdatasets/reaction-enthalpies.

\subsection{Calculation of errors}

To gauge the deviation of the theoretical reaction enthalpies from their experimental counterparts, we used the two common protocols based on the mean unsigned error (MUE $=\mid \Delta H($ exp. $)-$ $\Delta H($ theo. $) \mid)$ and the mean signed error (MSE $=\Delta H($ exp.) $\Delta H$ (theo.)). Simultaneous analysis of both MUE and MSE is necessary to make a conclusion on the ability of the method to provide the absolute reaction enthalpies and on its predictive ability. Clearly, if both MUE and MSE approach zero, then the method can be recommended for absolute values and thus predictions. This is the best scenario. If MUE is large and MSE is equally large (either positive or negative), then the method cannot be recommended for estimation of absolute reaction enthalpies. However, it still can be used for predictions, since in some instances trends are more important than absolute values. Finally, the worst scenario is large MUE and vanishing MSE, when the method cannot be recommended either for accurate absolute enthalpies or for the relative values.

\subsection{Core correlation options}

In the current work the influence of the core correlation settings on the reaction enthalpies was investigated. With the cc-pwCVnZ(-PP) correlation consistent basis sets the two frozen-core options were tested. In the first option, FC (frozen core), all non-ECP electrons were included in the SCF part and only non-noble gas electrons were included in the post-HF procedure. For example, for calcium, 20 electrons were included in the SCF treatment and only 2 electrons were included in the post-HF treatment ([Ar] configuration is frozen). For $\mathrm{Pb}$ the $6 \mathrm{~s}^{2} 6 \mathrm{p}^{2}$ electrons were 
Table 1 Experimental gas phase formation enthalpies used to build the reaction database

\begin{tabular}{|c|c|c|c|}
\hline Entry & Expt $\Delta H_{\mathrm{f}}(298.15)$ & $\begin{array}{l}\text { Av. expt } \Delta H_{\mathrm{f}}(298.15) \\
\left(\mathrm{kcal} \mathrm{mol}^{-1}\right)\end{array}$ & $\begin{array}{l}\text { Lit. } \operatorname{CCSD}(\mathrm{T}) \Delta H_{\mathrm{f}}(298.15)^{a} \\
\left(\text { kcal mol }^{-1}\right)\end{array}$ \\
\hline \multicolumn{4}{|c|}{ Group 1} \\
\hline $\mathrm{LiH}$ & $33.61 \pm 0.01 ;^{68} 33.4 \pm 0.4 ;^{81} 33.76^{77}$ & $33.6(-0.6 ;+0.2)$ & $(33.2 ; 33.3 \pm 0.1)^{82}$ \\
\hline $\mathrm{LiF}$ & $-81.45 \pm 2.0 ;^{68}-81.5 \pm 0.8 ;^{81}-80.9^{77}$ & $-81.3(-2.2 ; 1.8)$ & $(-81.4 ;-81.3 \pm 0.3)^{82}$ \\
\hline $\mathrm{LiCl}$ & $-46.78 \pm 3.011 i^{68}-46.3 \pm 1.0 ;^{81}-46.8^{77}$ & $-46.6(-3.2 ; 2.8)$ & $(-46.1 ;-45.7 \pm 0.4)^{82}$ \\
\hline $\mathrm{LiBr}$ & $-36.81 \pm 3.11 ;^{68}-36.1 \pm 1.0 ;^{81}-36.3^{77}$ & $-36.4(-3.5 ; 2.7)$ & \\
\hline LiI & $-21.8 \pm 2.0 ;^{68}-20.4 \pm 2.0 ;^{81}-20.7^{77}$ & $-21.0(-2.8 ; 2.6)$ & \\
\hline $\mathrm{NaF}$ & $-69.42 \pm 0.5 ;^{68}-70.54 \pm 1.0 i^{81}-70.1^{77}$ & $-70.0(-1.5 ; 1.1)$ & $-69.7^{82}$ \\
\hline $\mathrm{NaCl}$ & $-43.36 \pm 0.50 ;^{68}-43.4 \pm 0.72 ;^{81}-43.58^{77}$ & $-43.4(-0.7 ; 0.7)$ & $-43.1^{82}$ \\
\hline $\mathrm{NaBr}$ & $-34.4 \pm 0.50 ;^{68}-34.9 \pm 0.72 ;^{81}-36.1^{77}$ & $-35.1(-1.0 ; 1.2)$ & \\
\hline NaI & $-21.7 \pm 0.72 ;^{81}-18.1^{77}$ & $-19.9(-2.5 ; 1.8)$ & \\
\hline \multicolumn{4}{|c|}{ Group 2} \\
\hline $\mathrm{Be}$ & $77.44 \pm 1.20 ;^{68,83} 78.0^{77}$ & $77.7(-1.5 ;+0.9)$ & \\
\hline $\mathrm{BeF}_{2}$ & $-190.25 \pm 1.0 ;^{68}-189.7 ;^{76,77}-190.34 \pm 1.0^{83}$ & $-190.1(-1.2 ; 0.8)$ & $-190.1^{84}$ \\
\hline $\mathrm{BeCl}_{2}$ & $-86.09 \pm 2.51 ;^{68}-85.7 ;^{76,77}-86.36 \pm 0.8^{83}$ & $-86.1(-2.5 ;+2.5)$ & $-86.5^{84}$ \\
\hline $\mathrm{BeO}$ & $32.6 \pm 3.11 ;^{68} 32.01 \pm 2.63 ;^{83} 30.6^{77}$ & $31.7(-2.3 ;+4.0)$ & $30.1^{84}$ \\
\hline $\mathrm{Mg}$ & $35.16 \pm 0.19 ;^{68,83} 35.0 \pm 0.3^{77}$ & $35.1(-0.4 ;+0.2)$ & \\
\hline $\mathrm{MgF}_{2}$ & $-173.70 \pm 0.79 ;^{68}-176.03 \pm 1.2 ;^{83}-173.2 \pm 1.5^{77}$ & $-174.3(-2.9 ; 2.6)$ & $-174.4^{84}$ \\
\hline $\mathrm{MgCl}_{2}$ & $-93.8 \pm 0.50 ;^{68}-95.2 \pm 1.2 ;^{83}-95.7^{77}$ & $-94.9(-1.5 ; 1.6)$ & $-95.1^{84}$ \\
\hline $\mathrm{MgBr}_{2}$ & $-72.39 \pm 2.51 ;^{68}-72.1 \pm 3.6 ;^{83}-74.6 \pm 3^{77}$ & $-73.0(-4.6 ; 4.5)$ & \\
\hline $\mathrm{MgI}_{2}$ & $-38.3 \pm 2.51 ;^{68}-39.2 \pm 3.6 ;^{83}-41.0 ;-40.7^{77}$ & $-39.8(-3.0 ; 4.2)$ & \\
\hline $\mathrm{Ca}$ & $42.50 \pm 0.20 ;^{68,83} 42.6 \pm 0.4^{77}$ & $42.6(-0.4 ; 0.4)$ & \\
\hline $\mathrm{CaF}_{2}$ & $-187.5 \pm 1.91 ;^{68}-186.3 \pm 3.0 ;^{76,77}-189.0 \pm 1.6 ;^{83}-186.8^{77}$ & $-187.4(-3.2 ; 4.1)$ & $-189.1^{84}$ \\
\hline $\mathrm{CaBr}_{2}$ & $-92 \pm 2.0 ;^{68}-93.5 \pm 3 ;^{76,77}-92.83 \pm 2.2 ;^{83}-94.6 ;^{77}-92.5^{77}$ & $-93.1(-3.4 ;+3.1)$ & \\
\hline & $39.20 \pm 0.41 ;^{68} 38.36 \pm 0.48 ;^{83} 39.1^{77}$ & $38.9(-1.0 ; 0.7)$ & \\
\hline $\mathrm{SrF}_{2}$ & & $-184.4(-4.8 ; 2.3)$ & \\
\hline $\mathrm{SrCl}_{2}$ & $-183.1 \pm 1.00 ;^{68}-187.57 \pm 1.60 ;^{83}-182.7 ;-186.0^{77}$ & $-114.6(-3.4 ; 3.0)$ & \\
\hline $\mathrm{SrBr}_{2}$ & $-113.09 \pm 1.51 ;^{68}-112.6 ;^{76,77}-116.4 \pm 1.60 ;^{83}-114.9 ;-116.1^{77}$ & $-98.1(-2.9 ; 4.3)$ & \\
\hline $\mathrm{SrI}_{2}$ & $-97.30 \pm 3.01 ;^{68}-96.01 \pm 2.63 ;^{83}-98 ;^{77}-101.0^{76,77}$ & $-65.1(-2.9 ; 2.9)$ & \\
\hline $\mathrm{Ba}$ & $-65.70 \pm 1.51 ;^{68}-65.1 \pm 2.87 ;^{83}-65 ;^{77}-64.7^{77}$ & $43.1(-1.5 ;+0.9)$ & \\
\hline $\mathrm{BaF}_{2}$ & $42.81 \pm 1.20 ;^{68} 42.78 \pm 1.0 ;^{83} 43.8^{77}$ & $-192.2(-1.8 ;+3.6)$ & \\
\hline $\mathrm{BaCl}_{2}$ & $-192.10 \pm 1.51 ;^{68}-191.21 \pm 2.7 ;^{83}-192.5^{77}$ & $-118.4(-3.1 ;+4)$ & \\
\hline $\mathrm{BaO}$ & $-119.20 \pm 1.6 ;^{68}-116.7 \pm 2.3 ;^{83}-119.5 \pm 1 ;-118.3^{77}$ & $-30.4(-2.0 ;+2.9)$ & \\
\hline \multicolumn{4}{|l|}{$\mathrm{Pb}$ (II) } \\
\hline $\mathrm{PbCl}_{2}$ & $-41.6 \pm 0.3 ;^{68}-41.5 \pm 1.0 ;^{76,77}-42.0 \pm 1.2^{78}$ & $-41.7(-1.5 ; 1.2)$ & $-44.9^{80}$ \\
\hline $\mathrm{PbBr}_{2}$ & $-24.95 \pm 1.5 ;^{68}-25.2^{76,77}$ & $-25.1(-1.4 ; 1.6)$ & \\
\hline $\mathrm{PbI}_{2}$ & $-0.76 \pm 1.0 ;^{68}-2.08 \pm 1.2^{76,77}$ & $-1.4(-1.9 ; 1.6)$ & \\
\hline \multicolumn{4}{|c|}{ Complexes to equilibrate reactions } \\
\hline $\mathrm{H}_{2}$ & 0 & & \\
\hline $\mathrm{F}_{2}$ & 0 & & \\
\hline $\mathrm{Cl}_{2}$ & 0 & & \\
\hline $\mathrm{Br}_{2}$ & $7.388 \pm 0.026^{68}$ & & \\
\hline $\mathrm{I}_{2}$ & $14.92 \pm 0.019^{68}$ & & \\
\hline $\mathrm{H}_{2} \mathrm{O}$ & $-57.799 \pm 0.01^{68}$ & & \\
\hline $\mathrm{HF}$ & $-65.14 \pm 0.19^{68}$ & & \\
\hline $\mathrm{HCl}$ & $-22.06 \pm 0.05^{68}$ & & \\
\hline $\mathrm{HBr}$ & $-8.71 \pm 0.04^{68}$ & & \\
\hline HI & $6.30 \pm 0.05^{68}$ & & \\
\hline
\end{tabular}

treated as valence, i.e. included in the correlation treatment, while $5 s^{2} 5 p^{6} 5 d^{10}$ electrons were frozen. In the second option, $\mathrm{AE}$ (all electron), all non-ECP electrons (e.g. 20 for calcium) were included in both the SCF and correlation treatment. The summary of these two methods can be found in Table 3 .

Obviously, the AE protocol is computationally more demanding while the FC protocol is the most computationally effective. Which protocol provides the best accuracy/CPU cost ratio will be investigated in the current work.

\subsection{Selection of core orbitals}

By default, molecular orbitals (MOs) are ordered with respect to their orbital energies. The $\mathrm{N}$ lowest energy MOs, where $\mathrm{N}$ is the sum of core orbitals over all atoms, define the core region. All MOs with higher orbital energy define the valence region. If frozen core settings are not chosen carefully, the default ordering can lead to inversion of (physical) core and valence orbitals. A routine was implemented that detects and repairs inversion of core and valence orbitals and detects mixing of core orbitals with core or valence orbitals. The routine is described in the following.

First, for each atom type, the sum of core orbitals is calculated, based on the number of frozen core electrons per atom type. Next, for each MO, the Mulliken population per atom and angular momentum is computed. Each MO is then assigned to the atom type that has maximum Mulliken population. All MOs are then investigated in the order of their orbital energy, starting with 
the lowest energy MO. A MO is considered a core MO if the sum of core orbitals for its parent atom type has not yet been reached. All other MOs are considered valence MOs. After this assignment it is checked whether MOs that are found in the core region are considered valence MOs and whether MOs that are found in the valence region are considered core MOs. If such a case is found, it is corrected by inverting the ordering of the respective MOs. If a MO is considered a core MO and if only less than $90 \%$ of its Mulliken population is assigned to one combination of atom type and angular momentum, then a warning is printed that significant mixing is present. If such warning on orbital mixing is detected, the calculation might optionally be stopped just after initial (HF) wave function analysis to prevent subsequent computationally expensive post-HF part.

\section{Results and discussion}

\subsection{Benchmark of the basis sets/auxiliary basis sets}

A wide range of basis sets have been optimized and extensively tested in conjunction with highly correlated methods and the FC approach. On the other hand, in this context, basis sets for AE calculations are by far not that common and are much less tested. The same problem occurs for the auxiliary basis set to be used in the DLPNO calculations.

In this section, a preliminary study on the performance of Ahlrichs, Sapporo and correlation consistent basis set families for all-electron CCSD calculations has been carried out. For each family, the performance of triple- and quadruple- $\zeta$ basis sets in describing the different components of the correlation energy has been tested (see Table 4 for a summary of the basis sets used and of the corresponding labels). The effect of the auxiliary basis set to be used in the DLPNO-CCSD case has also been investigated.

A prerequisite for a suitable description of core-correlation effects in a chemical process is the accurate treatment of the core-core (cc), core-valence (cv) and valence-valence (vv) components of the correlation energy for the atoms involved in the reaction. Therefore, as a first prototype study case, we report in the following the basis set convergence behaviour of the different contributions of the CCSD correlation energy for the $\mathrm{Ca}$ atom. The results are summarized in Fig. 1.

In all cases, extrapolated values from correlation consistent basis sets (CCBS, see Table 4) have been used as reference (see Section 2.2.3 for details about the extrapolation technique). In this study, core and valence electrons have been defined in accordance with the so-called "chemical-core" used in ORCA 3.0 in the frozen-core approach, ${ }^{87}$ and the correlation energy has been divided accordingly. Therefore, all electrons are labelled as "core" but the ones in the outermost $4 \mathrm{~s}$ orbital.

As expected, the largest deviation is in the core-core correlation energy for all basis sets (upper panel of Fig. 1), while the remaining contributions are comparatively much closer to the CCBS reference. The Ahlrichs' and correlation consistent basis sets provide very similar performance and the inclusion of Peterson's weighted core-valence functions significantly improves the results
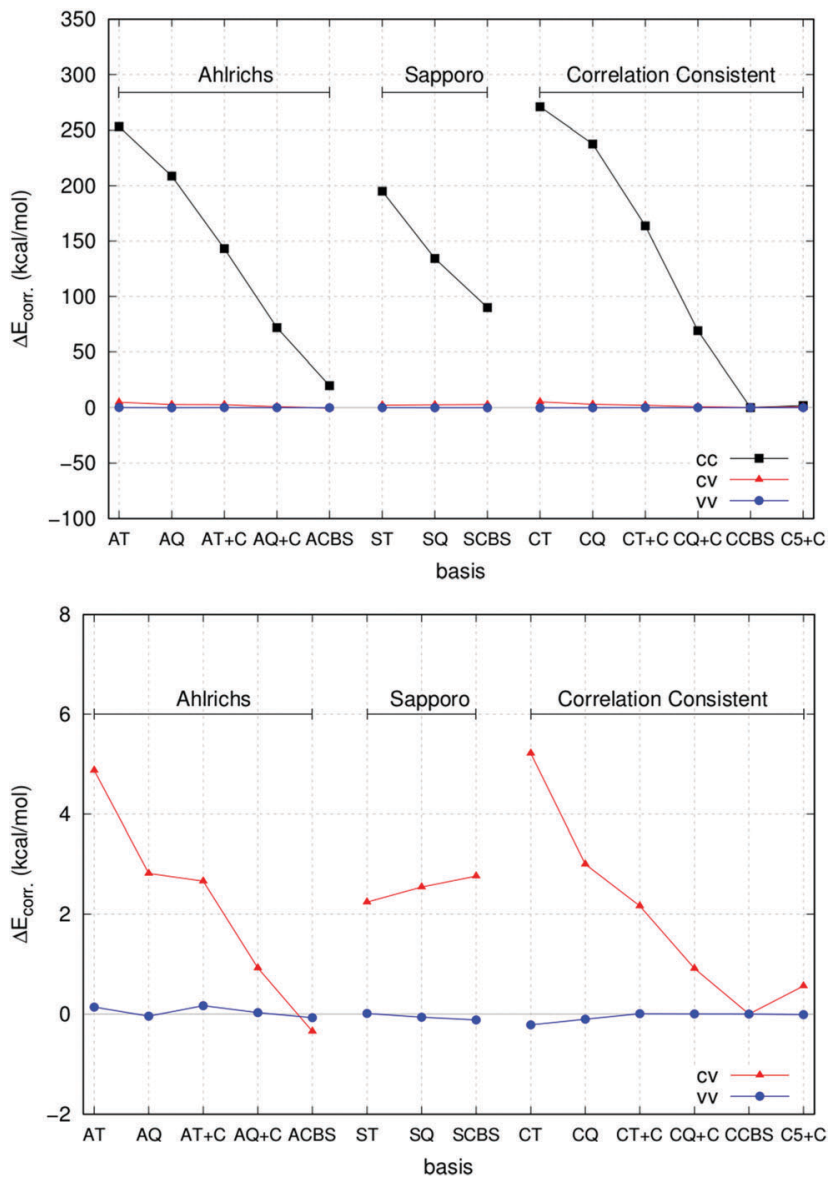

Fig. 1 (upper panel) Core-core (cc), core-valence (cv) and valencevalence (vv) correlation energy contributions for the calcium atom computed using different basis sets. Extrapolated values from the correlation consistent basis set are used as reference (CCBS). See text for details. (lower panel) The cv and vv contributions are plotted on a smaller scale for an easier comparison.

in both cases. Remarkably enough, the very large cc-pwCV5Z basis set gives values that are very close to the CCBS reference. This is true also for the core-valence and valence-valence components of the correlation energy, as shown in the lower panel of Fig. 1. Interestingly, the Sapporo basis sets provide much smaller correlation contributions and will not be used for routine calculations in this work.

These preliminary results suggest to test the performance of correlation consistent basis sets, in conjunction with the extrapolation scheme described in Section 2.2.2, on a wider range of situations (see Section 3.2). We recall here that, for most of the atoms studied in this work, these basis sets are used in conjunction with small-core pseudopotentials (the cc-pwCVNZ-PP set, see Section 2.2.1 for details). It is worth mentioning that in ccpwCVnZ basis sets for $\mathrm{Na}, \mathrm{Mg}, \mathrm{Cl}$ and $\mathrm{Ca}$, the deep core electrons, namely $1 \mathrm{~s}$ for $\mathrm{Na}, \mathrm{Mg}$ and $\mathrm{Cl}$ and $1 \mathrm{~s} 2 \mathrm{~s} 2 \mathrm{p}$ for $\mathrm{Ca}$, are described with single contracted Gaussian functions not directly optimized to be used in calculations including electron correlation. Therefore, the use of this family of basis sets in AE calculations is, in principle, not absolutely rigorous. However, we expect that the error introduced by using cc-pwCVnZ basis sets in AE calculations is small as deep 
core electrons are inert and do not participate in chemical bonding. Indeed, additional calculations performed with the "reduced" frozen core approximation, in which in addition to all valence electrons only the sub-valence (not deep core) electrons on the metal were correlated, resulted in reaction enthalpies very close to those from the $\mathrm{AE}$ calculations in which all the electrons were correlated (vide infra).

As already mentioned, the DLPNO-CCSD method relies on the RI approximation, and the quality of the auxiliary basis set used in the calculation strongly affects the overall accuracy. In general, the auxiliary basis sets def2-tzvpp/C and def2-qzvpp/C, available for most elements, perform well and can be used in conjunction with the corresponding triple- and quadruple- $\zeta$ basis sets. However, the accuracy of these auxiliary sets in conjunction with Peterson's basis sets in all-electron calculations needs to be tested.

As a first example, we initially computed cc, cv and vv correlation energy contributions for the $\mathrm{Ca}$ atom at the all electron DLPNO-CCSD level using different auxiliary basis sets and TCutPNO $=0$. With this TCutPNO threshold, DLPNO-CCSD and canonical CCSD are expected to give similar absolute energies, provided that the auxiliary basis set is good enough. Fig. 2 shows the error in the DLPNO-CCSD correlation energy terms with respect to canonical CCSD for different auxiliary basis sets.

For this atom, the error in the absolute energy between DLPNO-CCSD and canonical CCSD is very small with all auxiliary basis sets (note that relative energies are typically two orders of magnitude more accurate than absolute energies due to error cancellation). However, the error associated with the def2-tzvpp/C basis set in conjunction with cc-pwCVTZ is comparatively much larger. For this reason, the def2-qzvpp/C basis set will be used in the following in conjunction with both the cc-pwCVTZ and cc-pwCVQZ basis sets.

\subsection{Statistical study}

This section is organized as follows. First, the performance of the $\operatorname{CCSD}(\mathrm{T})$ method in its frozen core and all electron approaches in

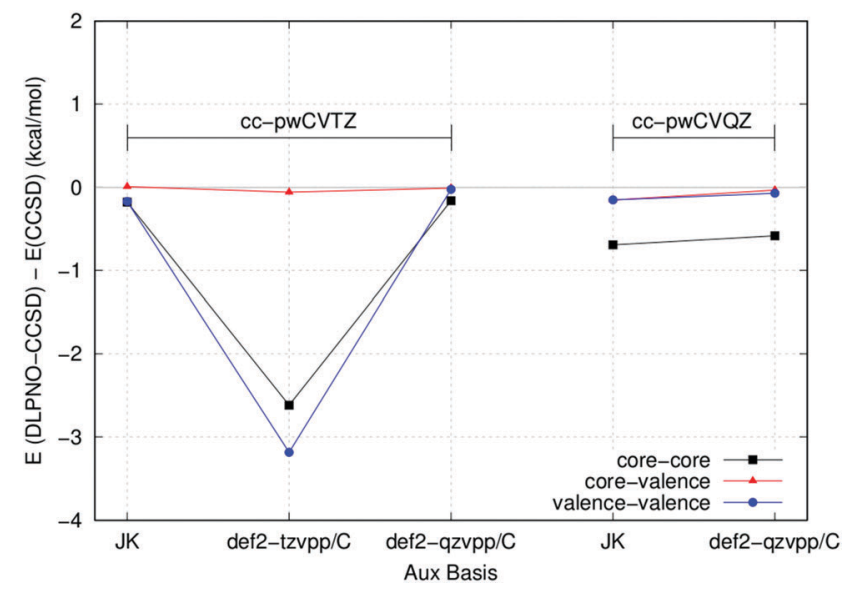

Fig. 2 Absolute error in the $c c, c v$ and vv correlation energy contributions with different auxiliary basis sets for the Ca atom and cc-pwCVTZ/QZ basis sets. Canonical CCSD energies are used as reference. reproducing 9 reaction enthalpies involving 2 nd period elements ( $\mathrm{Li}, \mathrm{Be}$ ) is discussed. Second, we proceed to 11 reaction enthalpies of complexes of 3rd period elements ( $\mathrm{Na}, \mathrm{Mg}$ ). After that we concentrate on 6 reaction enthalpies involving $\mathrm{Ca}$ and $\mathrm{Sr}$ complexes ( 2 group, 4 th and 5 th periods). Next, we comment on the performance obtained for 6 th period heavy elements, $\mathrm{Ba}$ and $\mathrm{Pb}$ (II). Then, we discuss the overall performance obtained for the combined dataset involving all the 8 non-transition metals. Practical recommendations on the frozen core approach for chemistry of non-transition metals will be given.

3.2.1 Second period ( $\mathbf{L i}, \mathrm{Be})$ complexes. The deviations obtained for reaction enthalpies involving $\mathrm{Li}$ and Be complexes calculated with correlation consistent basis sets and $\operatorname{CCSD}(\mathrm{T})$ and DLPNO-CCSD(T) methods and with the FC and AE approaches in the CBS extrapolation limit are presented in Chart 1. Encouragingly, both $\operatorname{CCSD}(\mathrm{T})$ and DLPNO-CCSD(T) methods with both FC and AE schemes provide absolute errors smaller than $2.3 \mathrm{kcal} \mathrm{mol}^{-1}$ for all the reactions. The largest deviations of $2.3 \mathrm{kcal} \mathrm{mol}^{-1}$ have been obtained with the DLPNO-CCSD(T) method and the AE approach for reactions (5) and (6). However, the deviation for reaction (6) is within the corresponding experimental uncertainty and for reaction (5) it is only $0.1 \mathrm{kcal} \mathrm{mol}^{-1}$ larger (see Table 2).

In general, the FC approach leads to somewhat smaller errors compared to the $\mathrm{AE}$ approach. In particular, for reactions (1), (5) and (6), the deviations in the reaction enthalpies are smaller by $1.2,2.2$ and $1.5 \mathrm{kcal} \mathrm{mol}^{-1}$, respectively, when the FC approach is applied. This perhaps counterintuitive conclusion can be explained by the fact that the deviations obtained with both FC and AE approaches are quite small and almost within the associated experimental uncertainties. Therefore, no statistically sound conclusion on the better performance of the frozen core approach can be made.

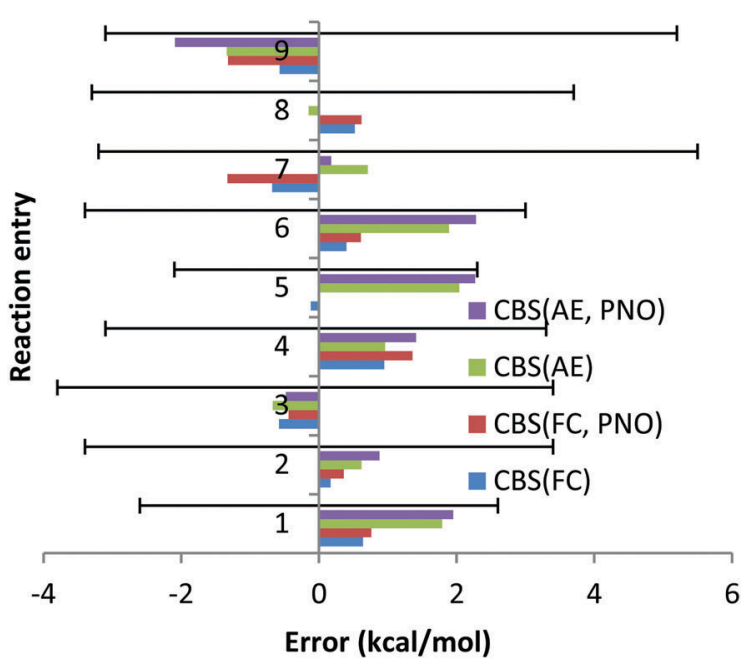

Chart 1 The deviations with respect to experimental reaction enthalpies obtained for the second period dataset $(\mathrm{Li}, \mathrm{Be})$ with correlation consistent basis sets in CBS TZ/QZ extrapolation and canonical CCSD(T) and DLPNO$\operatorname{CCSD}(T)$ methods and with FC ("frozen core") and AE ("all electron") approaches. The experimental deviations for each reaction are represented by black solid lines. 
Table 21 and 2 group metals and $\mathrm{Pb}(॥)$ gas phase reaction enthalpies used to build the datasets

\begin{tabular}{|c|c|c|}
\hline No. & Reaction & Expt avg. $\Delta H^{\circ}(298.15)\left(\mathrm{kcal} \mathrm{mol}^{-1}\right)$ \\
\hline 1 & $\mathrm{LiH}+\mathrm{F}_{2}=\mathrm{LiF}+\mathrm{HF}$ & $-180.0(-2.6 ; 2.6)$ \\
\hline 2 & $\mathrm{LiH}+\mathrm{Cl}_{2}=\mathrm{LiCl}+\mathrm{HCl}$ & $-102.3(-3.4 ; 3.4)$ \\
\hline 3 & $\mathrm{LiH}+\mathrm{Br}_{2}=\mathrm{LiBr}+\mathrm{HBr}$ & $-86.1(-3.8 ; 3.4)$ \\
\hline 4 & $\mathrm{LiH}+\mathrm{I}_{2}=\mathrm{LiI}_{2}+\mathrm{HI}$ & $-63.2(-3.1 ; 3.3)$ \\
\hline 5 & $\mathrm{Be}+\mathrm{F}_{2}=\mathrm{BeF}_{2}$ & $-267.8(-2.1 ; 2.3)$ \\
\hline 6 & $\mathrm{Be}+\mathrm{Cl}_{2}=\mathrm{BeCl}_{2}$ & $-163.8(-3.4 ; 4.0)$ \\
\hline 7 & $\mathrm{Be}+\mathrm{H}_{2} \mathrm{O}=\mathrm{BeO}+\mathrm{H}_{2}$ & $11.8(-3.2 ; 5.5)$ \\
\hline 8 & $\mathrm{BeF}_{2}+\mathrm{Cl}_{2}=\mathrm{BeCl}_{2}+\mathrm{F}_{2}$ & $104.0(-3.3 ; 3.7)$ \\
\hline 9 & $\mathrm{BeF}_{2}+\mathrm{H}_{2} \mathrm{O}=\mathrm{BeO}+\mathrm{F}_{2}+\mathrm{H}_{2}$ & $279.6(-3.1 ; 5.2)$ \\
\hline 10 & $\mathrm{NaF}+\mathrm{HCl}=\mathrm{NaCl}+\mathrm{HF}$ & $-16.5(-2.0 ; 2.4)$ \\
\hline 11 & $\mathrm{NaF}+\mathrm{HBr}=\mathrm{NaBr}+\mathrm{HF}$ & $-21.5(-2.3 ; 2.9)$ \\
\hline 12 & $\mathrm{NaF}+\mathrm{HI}=\mathrm{NaI}+\mathrm{HF}$ & $-21.3(-3.9 ; 3.5)$ \\
\hline 13 & $\mathrm{NaCl}+\mathrm{HBr}=\mathrm{NaBr}+\mathrm{HCl}$ & $-5.1(-1.8 ; 2.0)$ \\
\hline 14 & $\mathrm{NaCl}+\mathrm{HI}=\mathrm{NaI}+\mathrm{HCl}$ & $-4.9(-3.3 ; 2.6)$ \\
\hline 15 & $\mathrm{NaBr}+\mathrm{HI}=\mathrm{NaI}+\mathrm{HBr}$ & $0.2(-3.8 ; 2.9)$ \\
\hline 16 & $\mathrm{Mg}+\mathrm{F}_{2}=\mathrm{MgF}_{2}$ & $-209.4(-3.1 ; 3.0)$ \\
\hline 17 & $\mathrm{Mg}+\mathrm{Cl}_{2}=\mathrm{MgCl}_{2}$ & $-130.0(-1.7 ; 2.0)$ \\
\hline 18 & $\mathrm{Mg}+\mathrm{Br}_{2}=\mathrm{MgBr}_{2}$ & $-115.5(-4.8 ; 4.9)$ \\
\hline 19 & $\mathrm{Mg}+\mathrm{I}_{2}=\mathrm{MgI}_{2}$ & $-89.8(-3.2 ; 4.6)$ \\
\hline 20 & $\mathrm{MgF}_{2}+\mathrm{Cl}_{2}=\mathrm{MgCl}_{2}+\mathrm{F}_{2}$ & $79.4(-4.1 ; 4.5)$ \\
\hline 21 & $\mathrm{Ca}+\mathrm{F}_{2}=\mathrm{CaF}_{2}$ & $-230.0(-3.6 ; 4.5)$ \\
\hline 22 & $\mathrm{Ca}+\mathrm{Br}_{2}=\mathrm{CaBr}_{2}$ & $-143.1(-3.8 ; 3.5)$ \\
\hline 23 & $\mathrm{Sr}+\mathrm{F}_{2}=\mathrm{SrF}_{2}$ & $-223.3(-5.5 ; 3.3)$ \\
\hline 24 & $\mathrm{Sr}+\mathrm{Cl}_{2}=\mathrm{SrCl}_{2}$ & $-153.5(-4.1 ; 4.0)$ \\
\hline 25 & $\mathrm{Sr}+\mathrm{Br}_{2}=\mathrm{SrBr}_{2}$ & $-144.4(-3.6 ; 5.3)$ \\
\hline 26 & $\mathrm{Sr}+\mathrm{I}_{2}=\mathrm{SrI}_{2}$ & $-118.9(-3.6 ; 3.9)$ \\
\hline 27 & $\mathrm{Ba}+\mathrm{F}_{2}=\mathrm{BaF}_{2}$ & $-235.2(-2.7 ; 5.2)$ \\
\hline 28 & $\mathrm{Ba}+\mathrm{Cl}_{2}=\mathrm{BaCl}_{2}$ & $-161.5(-4.0 ; 5.5)$ \\
\hline 29 & $\mathrm{Ba}+\mathrm{H}_{2} \mathrm{O}=\mathrm{BaO}+\mathrm{H}_{2}$ & $-15.9(-2.9 ; 4.4)$ \\
\hline 30 & $\mathrm{PbCl}_{2}+\mathrm{Br}_{2}=\mathrm{PbBr}_{2}+\mathrm{Cl}_{2}$ & $9.2(-2.6 ; 3.2)$ \\
\hline 31 & $\mathrm{PbCl}_{2}+\mathrm{I}_{2}=\mathrm{PbI}_{2}+\mathrm{Cl}_{2}$ & $25.4(-3.1 ; 3.1)$ \\
\hline 32 & $\mathrm{PbBr}_{2}+\mathrm{I}_{2}=\mathrm{PbI}_{2}+\mathrm{Br}_{2}$ & $16.2(-3.6 ; 3.0)$ \\
\hline
\end{tabular}

The excellent performance of the FC approach for the Li and Be reactions we studied suggests that no significant mixing between core and valence orbitals occurs and that all the core orbitals have been properly identified and isolated. In fact, only for one complex, $\mathrm{LiBr}$, inversion of the molecular orbitals was needed: MO 10, mainly consisting of the 1s core orbital of $\mathrm{Li}$, was replaced with MO 5, mainly consisting of the d orbital of $\mathrm{Br}$. This means that, apart from LiBr, simple energy-based selection of the core orbitals would be sufficient.

Then, as no artificial mixing of the core and valence orbitals has been detected, the magnitude of the core-core (cc) and core-valence (cv) correlation effects can be derived from the direct comparison of the FC and $\mathrm{AE}$ reaction enthalpies. Thus, for reactions (2)-(4), (8) and (9), the cc and cv correlation effects turned out to be smaller than $1 \mathrm{kcal} \mathrm{mol}^{-1}$. Significantly larger $\mathrm{cc}$ and cv correlation effects have been found for reactions (1), (5), (6) and (7). The largest effect of the core correlation of $2.4 \mathrm{kcal} \mathrm{mol}^{-1}$ has been found for reaction (5), indicating that inclusion of the 1s orbital of Be in the correlation is essential. This means that inclusion of the core correlation is important for accurate thermochemistry of $\mathrm{Li}$ and Be complexes.

The DLPNO truncation almost does not affect the absolute reaction enthalpies (see the last paragraph of Section 3.3.5), since the difference between canonical $\operatorname{CCSD}(\mathrm{T})$ and DLPNO values is only within a fraction of a kcal mol${ }^{-1}$. The largest deviation was obtained for reaction (9) and the AE approach, and amounts to $0.8 \mathrm{kcal} \mathrm{mol}^{-1}$ only.
Table 3 Core correlation options in combination with the basis sets used in the present work

\begin{tabular}{lll}
\hline Method & Orbitals in SCF & Orbitals in post-HF \\
\hline FC & All non-ECP & Noble gas ${ }^{a}$ \\
AE & All non-ECP & All non-ECP
\end{tabular}

${ }^{a}$ The noble gas core configuration is frozen and the corresponding orbitals are not included in the post-HF part. For example, for $\mathrm{Ca}$, the [Ar] configuration is frozen. For $\mathrm{Pb}$, the $6 \mathrm{~s}^{2} 6 \mathrm{p}^{2}$ electrons are treated as valence, i.e. included in the correlation treatment.

To have a more comprehensive vision, the overall performance obtained for the $9 \mathrm{Be}$ and Li reactions is given in Chart 2 for both canonical and DLPNO CCSD(T) (PNO) methods with the FC and AE approaches and with the TZ, QZ, 5Z (for DLPNO only) and CBS (TZ, QZ) and CBS (QZ, 5Z) extrapolation schemes. Analysis of Chart 2 indicates again a slightly better performance of the FC approach and a negligible difference between canonical and DLPNO CCSD(T) results.

Another difference between the $\mathrm{FC}$ and $\mathrm{AE}$ schemes is related to the convergence with respect to the basis set size. Regardless of the core correlation settings, a noticeable decrease in both MUE and MSE is observed when going from the TZ to QZ basis set, as expected. When going from the QZ to $5 \mathrm{Z}$ basis set, marginal differences in the MUE/MSE are observed, indicating that the results are essentially converged with the QZ basis set. However, both TZ/QZ and QZ/5Z CBS extrapolation schemes lead to smaller errors compared to the QZ results only in the case of the FC approach. In the case of the AE scheme, even a slight increase of errors is observed compared to the QZ results. Noteworthily, the CBS extrapolation based on TZ/QZ basis sets results almost in the same MUE/MSE compared to the QZ/5Z scheme, requiring quite demanding $5 \mathrm{Z}$ calculations.

3.2.2 Third period (Na, Mg) complexes. Deviations obtained for reaction enthalpies involving $\mathrm{Na}$ and $\mathrm{Mg}$ complexes calculated with $\operatorname{CCSD}(\mathrm{T})$ and DLPNO-CCSD(T) methods and with the FC and $\mathrm{AE}$ approaches in CBS extrapolation are presented in Chart 3. Compared to $\mathrm{Li}$ and Be complexes, significantly larger deviations have been obtained especially with the FC approximation. Thus, deviations larger than $3 \mathrm{kcal} \mathrm{mol}^{-1}$ have been obtained for reactions (12), (16), (17) and (19).

For reaction (12) the experimental uncertainty is $(-3.9$; 3.5) $\mathrm{kcal} \mathrm{mol}^{-1}$. The $\operatorname{CCSD}(\mathrm{T}) / \mathrm{CBS}$ method with the $\mathrm{AE}$ and FC approaches results in deviations of 2.2 and $3.9 \mathrm{kcal} \mathrm{mol}^{-1}$, respectively, which are almost within the experimental uncertainty. Similar conclusions can be drawn for reaction (19) for which the experimental uncertainty $(-3.2 ; 4.6)$ is comparable with the deviations obtained with the $\operatorname{CCSD}(\mathrm{T}) / \mathrm{CBS}$ scheme within the AE and FC approaches, 5.1 and $3.3 \mathrm{kcal} \mathrm{mol}^{-1}$.

For reactions (16) and (17) the deviations obtained with the $\operatorname{CCSD}(\mathrm{T}) / \mathrm{CBS}$ scheme within the FC approach of 4.1 and $3.9 \mathrm{kcal} \mathrm{mol}^{-1}$, respectively, turned out to be significantly larger compared to experimental uncertainties of $(-3.1 ; 3)$ and $(-1.7 ; 2.0)$ for these reactions. Meanwhile, the AE deviations are almost within the experimental uncertainties.

Inversion of the orbitals was required for complexes $\mathrm{MgBr}_{2}$, $\mathrm{NaBr}$ and $\mathrm{NaI}$. In all cases the $2 \mathrm{p}$ core orbitals of $\mathrm{Na}$ and $\mathrm{Mg}$ 
Table 4 Calcium basis sets and labels used

\begin{tabular}{|c|c|}
\hline Label & Common name \\
\hline $\begin{array}{l}\text { Correlation consistent basis } \text { set }^{54} \\
\text { CT } \\
\text { CQ } \\
\text { CT + C } \\
\text { CQ + C } \\
\text { CCBS } \\
\text { C5 + C }\end{array}$ & 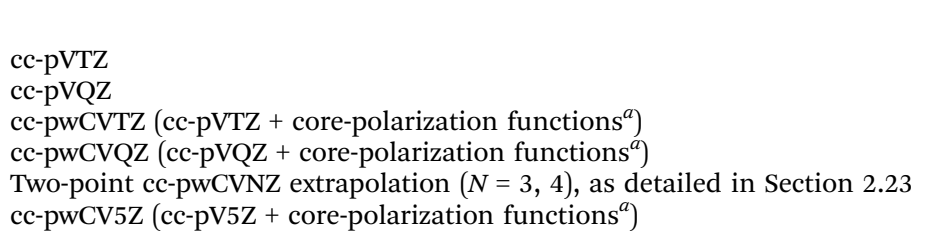 \\
\hline
\end{tabular}

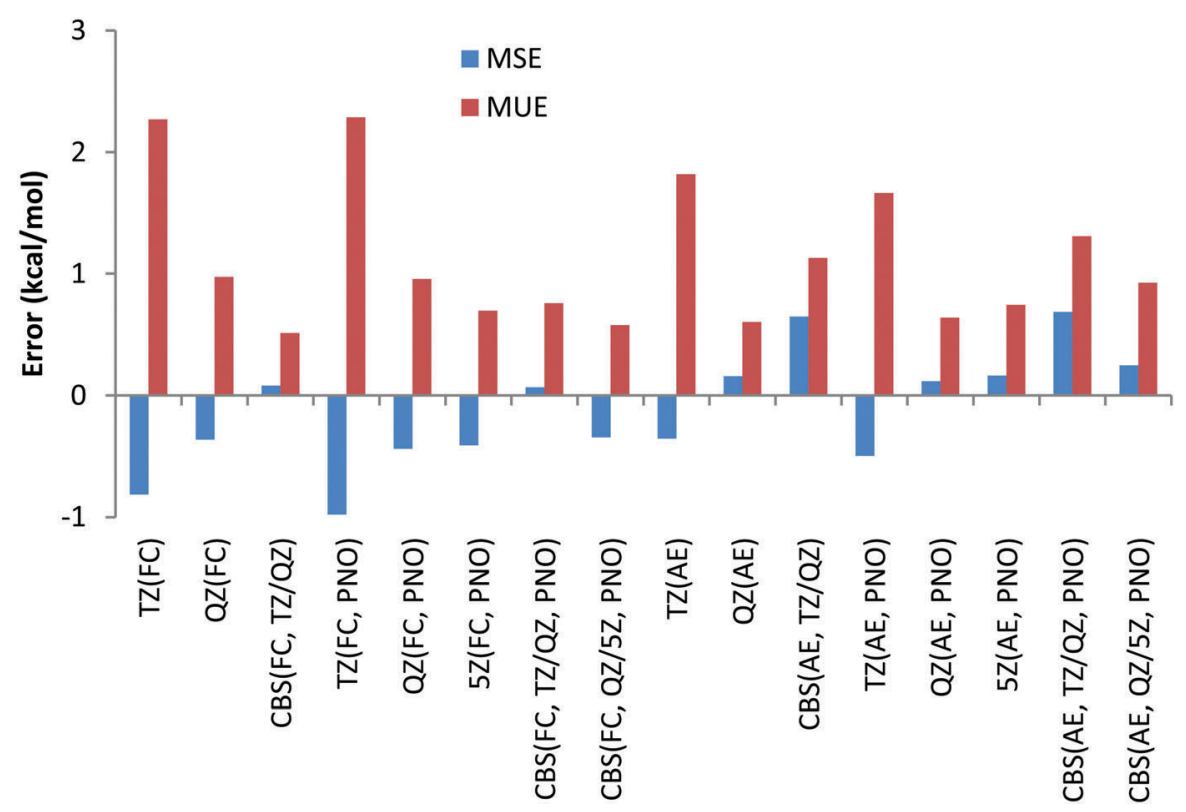

Chart 2 Mean unsigned (MUE) and mean signed (MSE) deviations with respect to experimental reaction enthalpies obtained for the second period dataset (Li, Be) with correlation consistent basis sets and canonical CCSD(T) and DLPNO-CCSD(T) methods and with FC ("frozen core") and AE ("all electron") approaches.

turned out to be in the valence region. No significant mixing between core and valence orbitals has been revealed for $\mathrm{Na}$ and $\mathrm{Mg}$ complexes studied in this work, with all the core orbitals properly identified and isolated. Therefore, comparison of the $\mathrm{FC}$ and $\mathrm{AE}$ results can be used to evaluate core-core and core-valence correlation effects. Only for two reactions, reactions (13) and (20), the cc and cv correlation effects were found to be smaller than $1 \mathrm{kcal} \mathrm{mol}^{-1}$. For all other reactions, these effects turned out to be significantly larger. The largest deviation between FC and $\mathrm{AE}$ approximations was found for reaction (18) and amounted to $2.3 \mathrm{kcal} \mathrm{mol}^{-1}$, indicating the importance of including core (sub-valence) orbitals in the calculations.

Thus, contrary to the Be and Li results, the FC approach in general results in larger deviations compared to the more CPU demanding $\mathrm{AE}$ scheme. This conclusion can also be drawn from Chart 4, where the overall performance obtained for the $11 \mathrm{Na}$ and $\mathrm{Mg}$ reactions is given for both canonical and DLPNO coupled cluster methods with FC and $\mathrm{AE}$ approaches and with TZ, QZ, 5Z and CBS TZ/QZ and CBS QZ/5Z (for DLPNO) extrapolation schemes. The poor performance of the FC approach for $\mathrm{Na}$ and $\mathrm{Mg}$ has also been emphasized previously by Martin $e t a .^{32}$ 


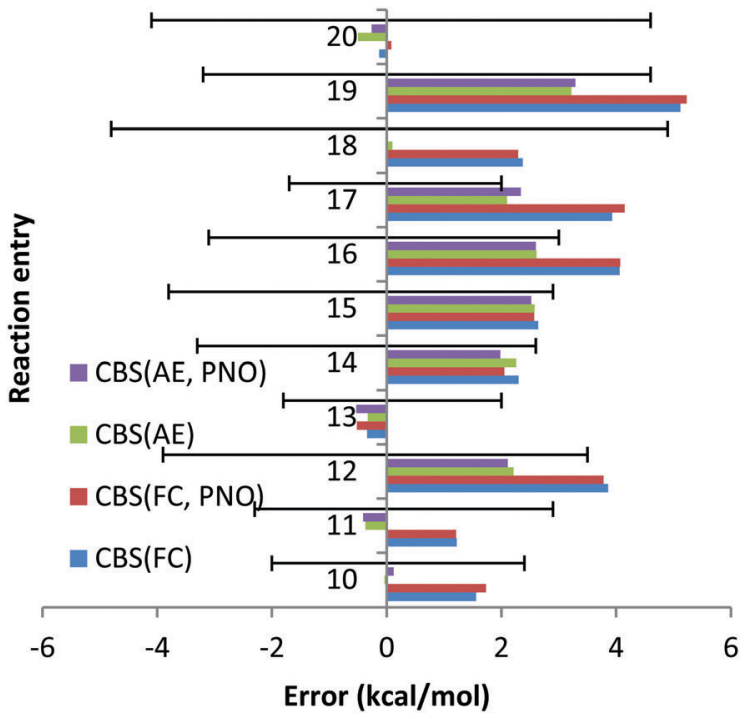

Chart 3 The deviations with respect to experimental reaction enthalpies obtained for the third period dataset $(\mathrm{Na}, \mathrm{Mg})$ with correlation consistent basis sets in CBS TZ/QZ extrapolation and canonical CCSD(T) and DLPNO$\operatorname{CCSD}(\mathrm{T})$ methods and with FC ("frozen core") and AE ("all electron") approaches. The experimental deviations for each reaction are represented by black solid lines.

Thus, CCSD(T)/CBS (FC) MUE is $2.5 \mathrm{kcal} \mathrm{mol}^{-1}$, which is $1 \mathrm{kcal} \mathrm{mol}^{-1}$ larger compared to $\mathrm{CCSD}(\mathrm{T}) / \mathrm{CBS}$ (AE) MUE. Another conclusion from the analysis of Chart 4 is that QZ results for all the method combinations provide the smallest deviations, making the use of relatively large $5 \mathrm{Z}$ basis sets and the CBS extrapolation schemes $\mathrm{TZ} / \mathrm{QZ}$ and $\mathrm{QZ} / 5 \mathrm{Z}$ redundant, at least for the reactions in this study. As previously found for Be and $\mathrm{Li}$ complexes, only a negligible difference, a fraction of a kcal mol ${ }^{-1}$, is found between DLPNO and canonical coupled cluster methods.

3.2.3 Fourth and fifth period $(\mathrm{Ca}, \mathrm{Sr})$ complexes. The deviations obtained for reaction enthalpies involving $\mathrm{Ca}$ and $\mathrm{Sr}$ complexes calculated with the correlation consistent basis set and canonical CCSD(T) and DLPNO-CCSD(T) methods and with the FC and AE approaches in CBS extrapolation are presented in Chart 5. Compared to the third period complexes, significantly larger deviations have been obtained. In particular, large deviations of more than $13 \mathrm{kcal} \mathrm{mol}^{-1}$ have been detected for reaction (21) when the FC was applied. Since that large deviation cannot be explained by the relatively modest experimental uncertainties of $(-3.6 ; 4.5) \mathrm{kcal} \mathrm{mol}^{-1}$ for this reaction, and because the $\mathrm{AE}$ approach performs significantly better, the FC approach is not applicable here. Indeed, it was already reported that neglecting (3s, $3 \mathrm{p}$ ) correlation in $\mathrm{K}$ and $\mathrm{Ca}$ compounds leads to erratic results at best, and chemically nonsensical ones if chalcogens or halogens are present. ${ }^{32}$ For this reaction the $\operatorname{CCSD}(\mathrm{T}) / \mathrm{CBS}(\mathrm{TZ} / \mathrm{QZ})$ AE approach yields a deviation equal to 6.9, which is $2.4 \mathrm{kcal} \mathrm{mol}^{-1}$ larger compared to the maximum experimental uncertainty.

However, the deviation obtained with the QZ basis set is only $1.1 \mathrm{kcal} \mathrm{mol}^{-1}$ (see Chart 6). At the same time, the $\operatorname{CCSD}(\mathrm{T}) / \mathrm{QZ} \mathrm{FC}$ MUE is $16.2 \mathrm{kcal} \mathrm{mol}^{-1}$, indicating clearly that the FC approach rather than the CBS extrapolation scheme is responsible for the failure. Interestingly, for reaction (23) the AE approach results in a deviation of $6.5 \mathrm{kcal} \mathrm{mol}^{-1}$, which is clearly larger than $2.4 \mathrm{kcal} \mathrm{mol}^{-1}$ obtained with the FC approach and cannot be explained by the experimental uncertainty of $(-5.5 ; 3.3)$. Once again, the $\operatorname{CCSD}(\mathrm{T}) / \mathrm{QZ}$ AE deviation is only $1.3 \mathrm{kcal} \mathrm{mol}^{-1}$ (see Chart 6), which is well below the experimental uncertainty, indicating that the CBS might result in the lack of some error cancellations taking place with the QZ basis set.

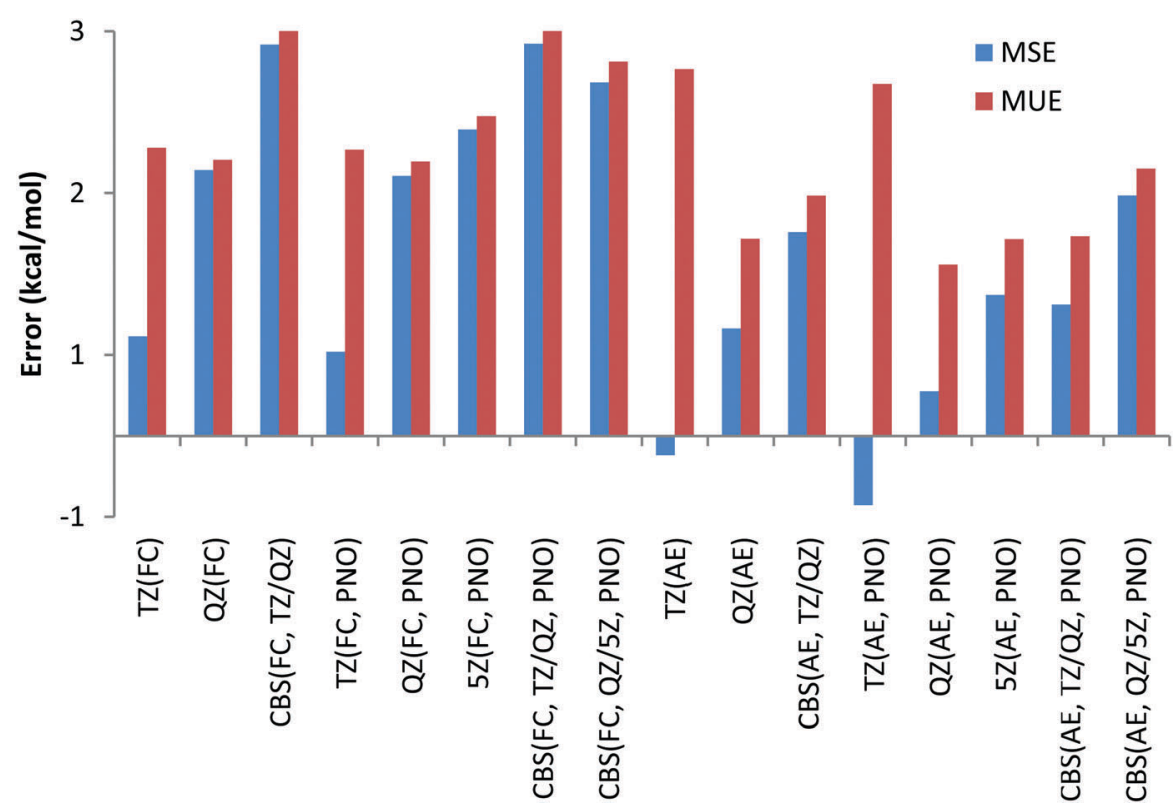

Chart 4 Mean unsigned (MUE) and mean signed (MSE) deviations with respect to experimental reaction enthalpies obtained for the third period dataset $(\mathrm{Na}, \mathrm{Mg})$ with correlation consistent basis sets and canonical CCSD(T) and DLPNO-CCSD(T) methods and with FC ("frozen core") and AE ("all electron") approaches. 


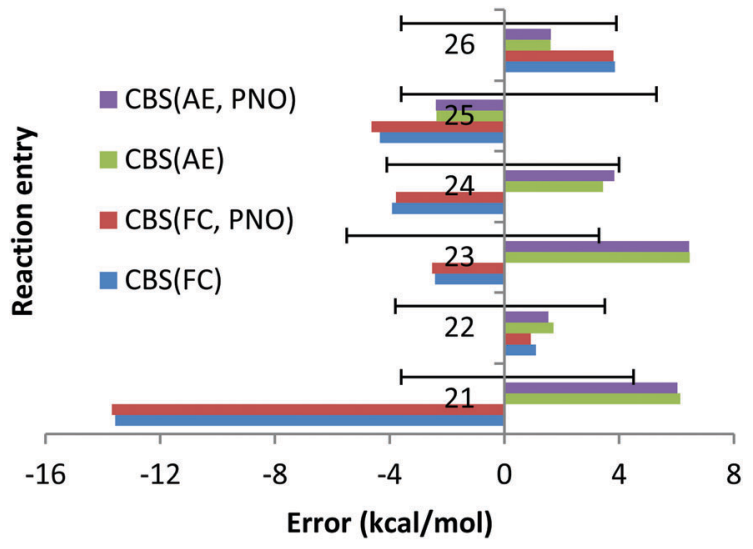

Chart 5 The deviations with respect to experimental reaction enthalpies obtained for the fourth and fifth period dataset $(\mathrm{Ca}, \mathrm{Sr})$ with correlation consistent basis sets in CBS TZ/QZ extrapolation and canonical CCSD(T) and DLPNO-CCSD(T) methods and with FC ("frozen core") and AE ("all electron") approaches. The experimental deviations for each reaction are represented by black solid lines.

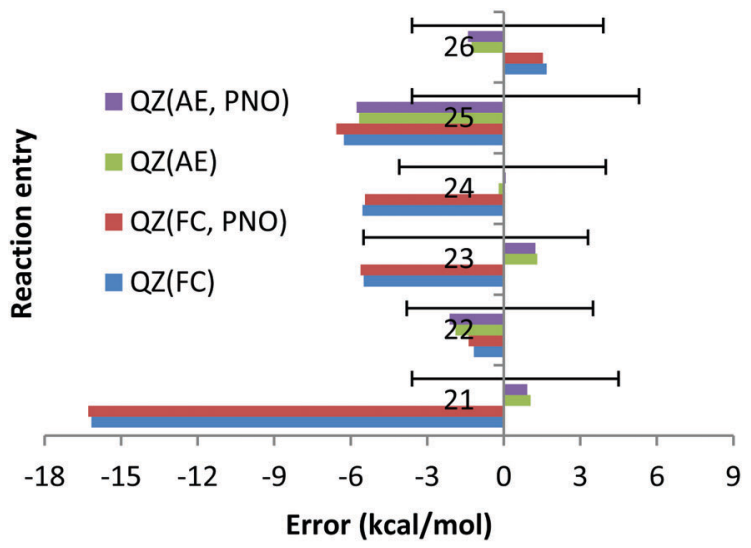

Chart 6 The deviations with respect to experimental reaction enthalpies obtained for the fourth and fifth period dataset $(\mathrm{Ca}, \mathrm{Sr})$ with correlation $\mathrm{QZ}$ consistent basis sets (cc-pwCVQZ(-PP)) and canonical CCSD(T) and DLPNO-CCSD(T) methods and with FC ("frozen core") and AE ("all electron") approaches. The experimental deviations for each reaction are represented by black solid lines.

In contrast to previous cases, apart from orbital inversion, significant mixing between core and valence orbitals has been found for two molecules of reactions (21)-(26). First, in $\mathrm{CaF}_{2}$, significant mixing occurs between the $3 \mathrm{p}$ core orbitals of $\mathrm{Ca}$ and the valence 2 s orbital of F (MO 13, see Fig. S1, ESI $\dagger$ ). Treating this orbital as core is apparently responsible for the large error documented for reaction (21), which is in line with previous works. $^{22,23}$ Indeed, additional calculations with the $3 \mathrm{~s}$ and $3 \mathrm{p}$ orbitals of $\mathrm{Ca}$ included in the correlation space and the 1s and 1s2s2p orbitals of F and Ca treated as a core ("reduced frozen core") resulted in a CBS reaction enthalpy for reaction (21) of $-234.7 \mathrm{kcal} \mathrm{mol}^{-1}$. This is in good agreement with the AE CBS enthalpy of $-236.1 \mathrm{kcal} \mathrm{mol}^{-1}$, but differs significantly from the FC CBS enthalpy of $-216.4 \mathrm{kcal} \mathrm{mol}^{-1}$. Second, mixing between the core $4 \mathrm{p}$ of $\mathrm{Sr}$ and the valence $3 \mathrm{~s}$ orbitals of $\mathrm{Cl}$ was found in $\mathrm{SrCl}_{2}$ (MO 12, see Fig. S2, ESI $\dagger$ ). However, in contrast to $\mathrm{CaF}_{2}$ the contribution of the $3 \mathrm{~s}$ valence orbital of the halogen is significantly smaller (the orbital is dominated by the $\mathrm{Sr} 4 \mathrm{p}$ orbital). Treating this orbital as core has resulted in the FC CBS enthalpy for reaction (24) of $-149.6 \mathrm{kcal} \mathrm{mol}^{-1}$, which is within the experimental uncertainties of the experimental enthalpy of $-153.5(-4.1 ; 4.0) \mathrm{kcal} \mathrm{mol}^{-1}$. However, still significant difference is observed upon comparison with the $\mathrm{AE}$ CBS enthalpy for this reaction, $-156.9 \mathrm{kcal} \mathrm{mol}^{-1}$. Although the difference is not as dramatic as for reaction (21), additional reduced frozen core calculations (with only the 4s4p orbitals of Sr moved to the correlation space) resulted in a CBS enthalpy for reaction (24) of $-156.7 \mathrm{kcal} \mathrm{mol}^{-1}$.

For $\mathrm{CaBr}_{2}$, no significant mixing between the core $3 \mathrm{~s} 3 \mathrm{p}$ orbitals of $\mathrm{Ca}$ and the valence $4 \mathrm{~s} 4 \mathrm{p}$ orbitals of $\mathrm{Br}$ was found. The FC and AE CBS enthalpies for reaction (22) turned out to be $-144.2 \mathrm{kcal} \mathrm{mol}^{-1}$ and $-144.8 \mathrm{kcal} \mathrm{mol}^{-1}$, indicating that the cc and cv effects are small. For $\mathrm{SrF}_{2}, \mathrm{SrBr}_{2}$ and $\mathrm{SrI}_{2}$, no mixing of the core $4 \mathrm{~s} 4 \mathrm{p}$ orbitals of $\mathrm{Sr}$ with the valence orbitals of halogens was found. However, large differences between the FC and AE reaction enthalpies have been obtained. Thus, for reaction (23) the FC CBS enthalpy is $-220.9 \mathrm{kcal} \mathrm{mol}^{-1}$, while the corresponding AE reaction enthalpy is $-229.8 \mathrm{kcal} \mathrm{mol}^{-1}$. This indicates that the $\mathrm{cc}$ and $\mathrm{cv}$ correlation effects are large for this reaction and amount to $9 \mathrm{kcal} \mathrm{mol}^{-1}$. We can rationalize this remarkable effect in terms of the dispersion interaction arising between the core electrons of $\mathrm{Sr}$ and the electrons on the $\mathrm{F}$ atoms. In fact, as already discussed by Petersson and co-workers, ${ }^{25}$ London dispersion forces between core electrons of one atom and electrons in neighbouring atoms can contribute significantly to binding energies. In the present case, the strong interaction between the $\mathrm{Sr}$ and the $\mathrm{F}$ atoms brings them very close to each other, leading to non-negligible attractive forces between their electrons. Indeed, reduced frozen core calculations with the $4 \mathrm{~s} 4 \mathrm{p}$ electrons of $\mathrm{Sr}$ included in the correlation space resulted in a CBS reaction enthalpy of $-229.3 \mathrm{kcal} \mathrm{mol}^{-1}$.

Similar comparisons made for reaction (25) have revealed the effects from the cc and cv correlation of $2 \mathrm{kcal} \mathrm{mol}^{-1}$. The $\mathrm{FC}, \mathrm{AE}$ and reduced frozen core $(4 \mathrm{~s} 4 \mathrm{p}$ of $\mathrm{Sr}$ moved to the correlation space) reaction enthalpies are $-140.1,-142.0$ and $-142.3 \mathrm{kcal} \mathrm{mol}^{-1}$, respectively. Finally, the FC, AE and reduced frozen core $(4 \mathrm{~s} 4 \mathrm{p}$ of $\mathrm{Sr}$ are correlated) reaction enthalpies are

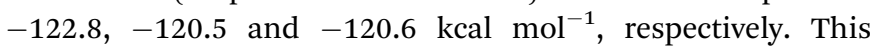
means that the cc and cv correlation effects are ca. $2 \mathrm{kcal} \mathrm{mol}^{-1}$ for the $\mathrm{SrI}_{2}$ reaction, indicating that even in the absence of core and valence orbital mixing, core-core and core-valence correlation effects can be very strong and cannot be ignored.

The smaller MUE/MSE values obtained with the QZ basis set are also confirmed in Chart 7 where the overall performance obtained for the $6 \mathrm{Ca}$ and $\mathrm{Sr}$ reactions is given for both canonical and DLPNO coupled cluster methods with the FC and AE approaches and with TZ, QZ, $5 Z$ and CBS TZ/QZ and QZ/5Z extrapolation schemes. Thus, MUE obtained with the QZ basis set is only $1.9 \mathrm{kcal} \mathrm{mol}^{-1}$ for the $\operatorname{CCSD}(\mathrm{T}) \mathrm{AE}$ method while the corresponding CBS value is $3.6 \mathrm{kcal} \mathrm{mol}^{-1}$, thus pointing to a questionable role of the CBS extrapolation scheme in this case. Also, application of large $5 \mathrm{Z}$ basis sets and the CBS 


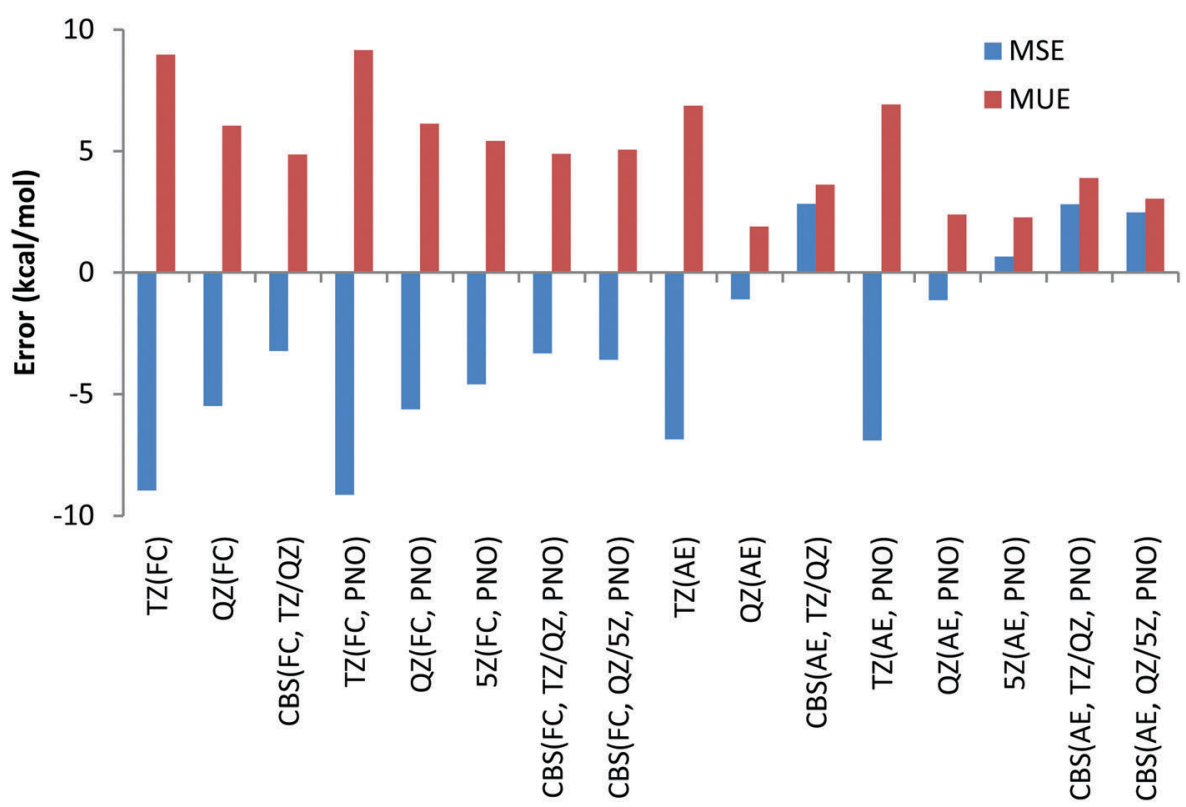

Chart 7 Mean unsigned (MUE) and mean signed (MSE) deviations with respect to experimental reaction enthalpies obtained for the fourth and fifth period dataset ( $\mathrm{Ca}$ and $\mathrm{Sr}$ ) with correlation consistent basis sets and canonical CCSD(T) and DLPNO-CCSD(T) methods and with FC ("frozen core") and AE ("all electron") approaches.

QZ/5Z extrapolation scheme does not improve the QZ performance, indicating that quite CPU demanding $5 \mathrm{Z}$ calculations can be avoided. The poor performance of the FC approach is also confirmed in Chart 7, where the left half of the chart clearly contains larger MUEs. As previously found, only negligible difference of a fraction of a kcal mol ${ }^{-1}$ between DLPNO and canonical coupled cluster methods can be detected.

3.2.4 Sixth period (Ba, $\mathbf{P b}(\mathrm{II}))$ complexes. Deviations obtained for reaction enthalpies involving $\mathrm{Ba}$ and $\mathrm{Pb}$ (II) complexes calculated with the correlation consistent basis set and CCSD(T) and DLPNO$\operatorname{CCSD}(\mathrm{T})$ methods and with the FC and $\mathrm{AE}$ approaches in the CBS extrapolation are presented in Chart 8. Similarly to the reactions of fourth and fifth period complexes, significant

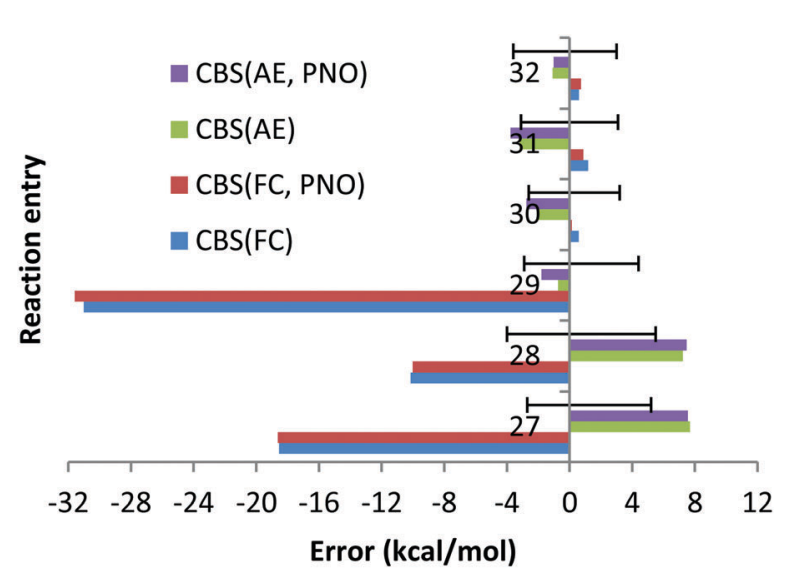

Chart 8 The deviations with respect to experimental reaction enthalpies obtained for the sixth period dataset $(\mathrm{Ba}, \mathrm{Pb}(\|))$ with correlation consistent basis sets in CBS extrapolation and $\operatorname{CCSD}(T)$ and DLPNO-CCSD(T) methods and with FC ("frozen core") and AE ("all electron") approaches. The experimental deviations for each reaction are represented by black solid lines. deviations $\left(\sim 10-32 \mathrm{kcal} \mathrm{mol}^{-1}\right)$ have been obtained with the FC approach. Since these large deviations cannot be explained by the relatively modest experimental uncertainties of 2-5 kcal mol${ }^{-1}$ and because the $\mathrm{AE}$ approach performs significantly better, the FC approach is not applicable for these reactions.

With the AE approach in the CBS limit the largest errors have been obtained for reactions (27) $\left(7.7 \mathrm{kcal} \mathrm{mol}^{-1}\right)$ and (28) $\left(7.2 \mathrm{kcal} \mathrm{mol}^{-1}\right)$. The quite large experimental uncertainties for those reactions $(-2.7 ; 5.2)$ and $(-4.0 ; 5.5) \mathrm{kcal} \mathrm{mol}^{-1}$, respectively, can partially account for such large deviations. The fact that deviations obtained with $\operatorname{CCSD}(\mathrm{T})$ and QZ basis sets and $\mathrm{AE}$ are only 1.3 and $2.7 \mathrm{kcal} \mathrm{mol}^{-1}$ (see Chart 9) would suggest that the

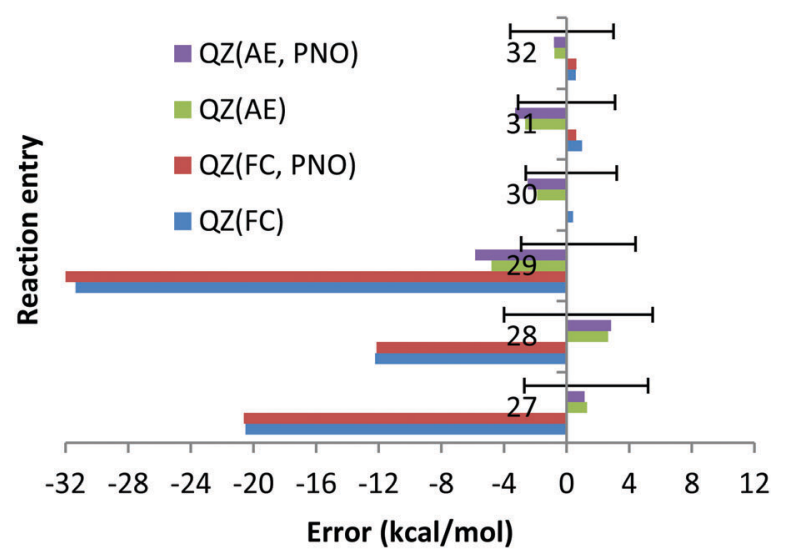

Chart 9 The deviations with respect to experimental reaction enthalpies obtained for the sixth period dataset $(\mathrm{Ba}, \mathrm{Pb}(\mathrm{II}))$ with correlation $\mathrm{QZ}$ consistent basis sets (cC-pwCVQZ(-PP)) and CCSD(T) and DLPNO$\operatorname{CCSD}(T)$ methods and with FC ("frozen core") and AE ("all electron") approaches. The experimental deviations for each reaction are represented by black solid lines. 
CBS extrapolation does not work for these two reactions. However, the fact that $5 \mathrm{Z}$ DLPNO-CCSD(T) AE results in deviations of 4.7 and $4.9 \mathrm{kcal} \mathrm{mol}^{-1}$ (QZ DLPNO-CCSD(T) errors are 1.9 and $3.6 \mathrm{kcal} \mathrm{mol}^{-1}$, again very close to canonical $\operatorname{CCSD}(\mathrm{T})$, see above and Chart 9) indicates that the CBS extrapolation scheme is not responsible for the large deviation, rather the errors are increasing upon the increase in the basis sets for these reactions in general, which is not as expected. Such strange behaviour of the $\operatorname{CCSD}(\mathrm{T})$ $\mathrm{AE}$ method for these two reactions might only be explained by the very difficult natures of these two reactions, since both of them are dissociation/association reactions $\left(\mathrm{Ba}+\mathrm{X}_{2}=\mathrm{BaX}_{2}, \mathrm{X}=\mathrm{F}, \mathrm{Cl}\right)$ where the metal changes the oxidation state from 0 (in the atom) to +2 and no error compensation can be expected. In addition, the electronic structure of barium is drastically different in both parts of the equations. We do not expect that the core orbitals (replaced by relativistic ECP on the Ba atom, e.g. as f-orbitals in the case of Hf complexes ${ }^{33}$ ) or approximation of the scalar relativistic effects by relativistic pseudopotential is responsible for these failures since the DLPNO-CCSD(T) method with QZ fully de-contracted Sapporo basis sets accomplished with diffuse functions for all the exponents and AutoAux auxiliary basis sets lead to deviations of 8.5 and $4.9 \mathrm{kcal} \mathrm{mol}^{-1}$ for reactions (27) and 28, respectively, which are almost identical to $\operatorname{CCSD}(\mathrm{T}) / \mathrm{CBS}$ AE results. Finally, it is not impossible that the experimental data are not very accurate for $\mathrm{BaF}_{2}$ and $\mathrm{BaCl}_{2}$.

The smaller MUE/MSE values obtained with the QZ basis set are also confirmed in Chart 10, where the overall performance obtained for the 6 reactions is given for both canonical and DLPNO coupled cluster methods with/without the frozen core approach and with TZ, QZ, 5Z and CBS TZ/QZ and QZ/5Z extrapolations. Thus, MUE obtained with the QZ basis set is only $2.3 \mathrm{kcal} \mathrm{mol} \mathrm{m}^{-1}$ for the $\operatorname{CCSD}(\mathrm{T}) \mathrm{AE}$ method while the corresponding CBS value is $3.7 \mathrm{kcal} \mathrm{mol}^{-1}$. The poor performance of the frozen core approach is also confirmed in Chart 10 (the errors in the left side of the chart are clearly higher). As previously found, only negligible difference of a few tenths of a kcal mol $\mathrm{m}^{-1}$ between DLPNO and canonical coupled cluster methods can be detected.

Strong mixing between the core $5 \mathrm{p}$ orbitals of $\mathrm{Ba}$ and the valence orbitals of oxygen and halogens was found for all the $\mathrm{Ba}$ compounds we studied, namely, $\mathrm{BaF}_{2}, \mathrm{BaCl}_{2}$ and $\mathrm{BaO}$. This indicates that the large errors observed for reactions (27)-(29) are due to inconsistent treatment of the core and valence correlation in $\mathrm{Ba}$. For $\mathrm{BaF}_{2}$, the mixing between the core (sub-valence) $5 \mathrm{~s}$ orbital of $\mathrm{Ba}$ and the $2 \mathrm{~s}$ valence orbital of $\mathrm{F}$ was identified in $\mathrm{MO}$ 3 (see Fig. S3 (ESI $\dagger$ )). Moreover, in MO 8 (Fig. S4, ESI $\dagger$ ), mixing between the core $5 \mathrm{p}$ orbital of $\mathrm{Ba}$ and the $2 \mathrm{~s} 2 \mathrm{p}$ valence orbitals of $\mathrm{F}$ was identified as well. The FC CBS predicted enthalpy of reaction (27) is $-216.7 \mathrm{kcal} \mathrm{mol}^{-1}$, versus an $\mathrm{AE}$ predicted enthalpy of $-242.9 \mathrm{kcal} \mathrm{mol}^{-1}$, indicating that inclusion of the sub-valence Ba $5 \mathrm{~s} 5 \mathrm{p}$ orbitals has a substantial effect. It is confirmed by reduced FC calculations (5s5p orbitals of Ba moved to the correlation space), in which the CBS enthalpy of reaction (27) is $-242.5 \mathrm{kcal} \mathrm{mol}^{-1}$. For $\mathrm{BaCl}_{2}$, significant mixing of the sub-valence (core) $5 \mathrm{p}$ orbitals of $\mathrm{Ba}$ with the $3 \mathrm{~s}$ valence orbital of $\mathrm{Cl}$ was identified in MO 16, treated as a core orbital (see Fig. S5 (ESI $\dagger$ )). The FC CBS predicted enthalpy of reaction (28) is $-151.4 \mathrm{kcal} \mathrm{mol}^{-1}$, versus an $\mathrm{AE}$ predicted enthalpy of $-168.7 \mathrm{kcal} \mathrm{mol}^{-1}$. Reduced FC calculations (5s5p orbitals of Ba moved to the correlation space) resulted in a CBS enthalpy of $-168.5 \mathrm{kcal} \mathrm{mol}^{-1}$, indicating that correlating the 5s5p orbitals is essential, while correlating the 1s2s2p orbitals of $\mathrm{Cl}$ is not. Finally, for $\mathrm{BaO}$, significant mixing of the core (sub-valence) Ba 5p orbital with the valence $\mathrm{O} 2 \mathrm{~s}$ orbital was identified in MO 6 (see Fig. S6 (ESI $\dagger$ )). The FC CBS predicted

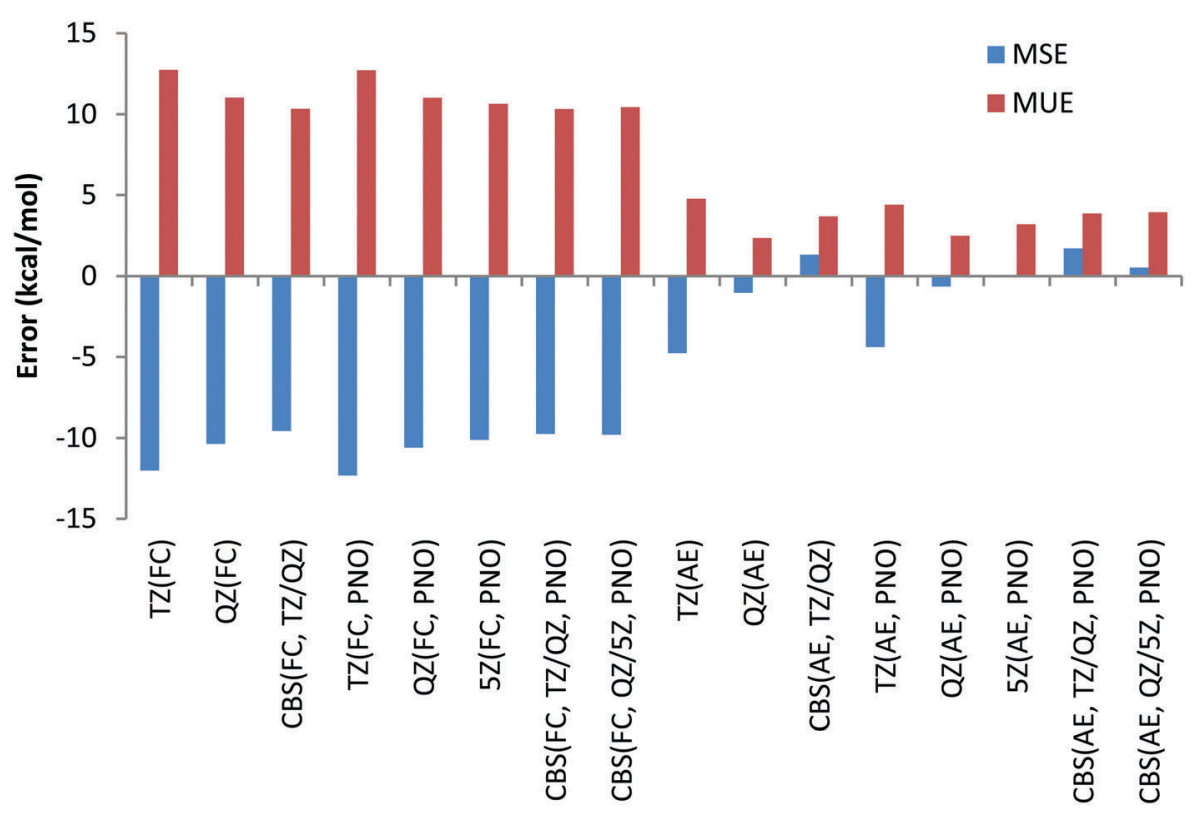

Chart 10 Mean unsigned (MUE) and mean signed (MSE) deviations with respect to experimental reaction enthalpies obtained for the sixth period dataset $(\mathrm{Ba}, \mathrm{Pb}(\mathrm{I}))$ with correlation consistent basis sets and canonical CCSD(T) and DLPNO-CCSD(T) and with FC ("frozen core") and AE ("all electron") approaches. 
enthalpy of reaction (28) is $15.1 \mathrm{kcal} \mathrm{mol}^{-1}$, versus an $\mathrm{AE}$ predicted enthalpy of $-15.2 \mathrm{kcal} \mathrm{mol}^{-1}$. Reduced FC calculations (5s5p orbitals of Ba moved to the correlation space) resulted in a CBS enthalpy of $-15.3 \mathrm{kcal} \mathrm{mol}^{-1}$, indicating that inclusion of the 5s5p Ba orbitals in the correlation space is essential (while inclusion of core 1 s orbitals of $\mathrm{O}$ is not).

No mixing of the core and valence orbitals has been found for the molecular core orbitals of the lead complexes we studied. Hence, the difference between the FC and the $\mathrm{AE}$ calculations can be attributed solely to the cc and cv correlation effects. The FC CBS enthalpy for reaction (30) is $8.6 \mathrm{kcal} \mathrm{mol}^{-1}$, versus an AE CBS enthalpy of $11.3 \mathrm{kcal} \mathrm{mol}^{-1}$, indicating that the cc and cv effect for this reaction is $2.7 \mathrm{kcal} \mathrm{mol}^{-1}$. Reduced FC calculations (5s5p of $\mathrm{Pb}$ moved to the correlation space) resulted in a CBS enthalpy of $11 \mathrm{kcal} \mathrm{mol}^{-1}$. The FC CBS enthalpy for reaction (31) is $24.2 \mathrm{kcal} \mathrm{mol}^{-1}$, versus an $\mathrm{AE}$ CBS enthalpy of $28.6 \mathrm{kcal} \mathrm{mol}^{-1}$, indicating that the cc and $\mathrm{cv}$ effect for this reaction is $4.4 \mathrm{kcal} \mathrm{mol}^{-1}$. Reduced FC calculations (5s5p orbitals of $\mathrm{Pb}$ included in the correlation space) resulted in a CBS enthalpy for this reaction of $28.5 \mathrm{kcal} \mathrm{mol}^{-1}$. The FC CBS enthalpy for reaction (32) is $15.6 \mathrm{kcal} \mathrm{mol}^{-1}$, versus an AE CBS enthalpy of 17.3, indicating that the cc and cv effect for this reaction is $1.7 \mathrm{kcal} \mathrm{mol}^{-1}$. Reduced FC calculations (5s5p orbitals of $\mathrm{Pb}$ moved to the correlation space) resulted in a CBS enthalpy for this reaction of $17.5 \mathrm{kcal} \mathrm{mol}^{-1}$. All these results indicate that inclusion of the sub-valence $5 \mathrm{~s} 5 \mathrm{p}$ orbitals of $\mathrm{Pb}$ in the correlation treatment is essential while inclusion of the core orbitals of halogens (1s for F, 1s $2 \mathrm{~s} 2 \mathrm{p}$ for $\mathrm{Cl}$ and $3 \mathrm{~s} 3 \mathrm{p}$ for $\mathrm{Br}$ ) is not.

3.2.5 Overall performance. The overall performance obtained for all 32 reactions of $\mathrm{Li}, \mathrm{Be}, \mathrm{Na}, \mathrm{Mg}, \mathrm{Sr}, \mathrm{Ba}$ and $\mathrm{Pb}$ complexes is given in Chart 11. As expected, due to large deviations obtained for the frozen core approach for some reactions of $\mathrm{Na}, \mathrm{Mg}, \mathrm{Ca}, \mathrm{Sr}$, and $\mathrm{Ba}$, the resulting FC MUEs are quite large (>3.9 $\mathrm{kcal} \mathrm{mol}^{-1}$ ) regardless of the method (canonical or DLPNO) and the basis sets. All the errors documented in the current work for the frozen core approximation can be classified into two groups: (a) errors due to mixing of the metal core orbitals with the valence orbitals of oxygen and halogens in the core MO orbitals; (b) errors exclusively due to neglecting core-core and core-valence correlation when no artificial mixing of the core and valence orbitals is observed. The largest errors obtained for the frozen core approximation are dominated by the first group. In particular, mixing of the core and valence orbitals has been identified for $\mathrm{CaF}_{2}, \mathrm{SrCl}_{2}, \mathrm{BaF}_{2}$, $\mathrm{BaCl}_{2}$ and $\mathrm{BaO}$, leading to errors up to $30-40 \mathrm{kcal} \mathrm{mol}^{-1}$ for some reactions. The second group of errors, dominated by neglecting cc and cv correlation, turned out to be essential for reactions involving $\mathrm{Pb}$ and $\mathrm{Sr}$ complexes. Comparison of the $\mathrm{FC}$ and $\mathrm{AE}$ reaction enthalpies indicated that $\mathrm{cc}$ and $\mathrm{cv}$ correlation is significant also for reactions involving $\mathrm{Li}, \mathrm{Be}, \mathrm{Na}$ and $\mathrm{Mg}$, and cannot be ignored if accurate reaction enthalpies are targeted. Both groups of errors can be ameliorated by extension of the correlation space with sub-valence orbitals of metals. More conservative FC defaults will be available in the upcoming ORCA 4.0 release. However, the new defaults will require careful basis set specifications: e.g. in the new FC default the $3 \mathrm{~s} 3 \mathrm{p}$ sub-valence orbitals of $\mathrm{Ca}$ are correlated and thus require cc-pwCVnZ basis sets on $\mathrm{Ca}$, since the standard cc-pVnZ basis set on Ca might result in errors as the inner $\mathrm{s}$ and $\mathrm{p}$ functions on Ca have not been designed to be correlated. Test calculations showed that the currently used chemical cores might be too large and that more conservative chemical core regions might significantly increase the accuracy, without the need to include all electrons in the correlation calculations. The chemical core regions for heavier elements will

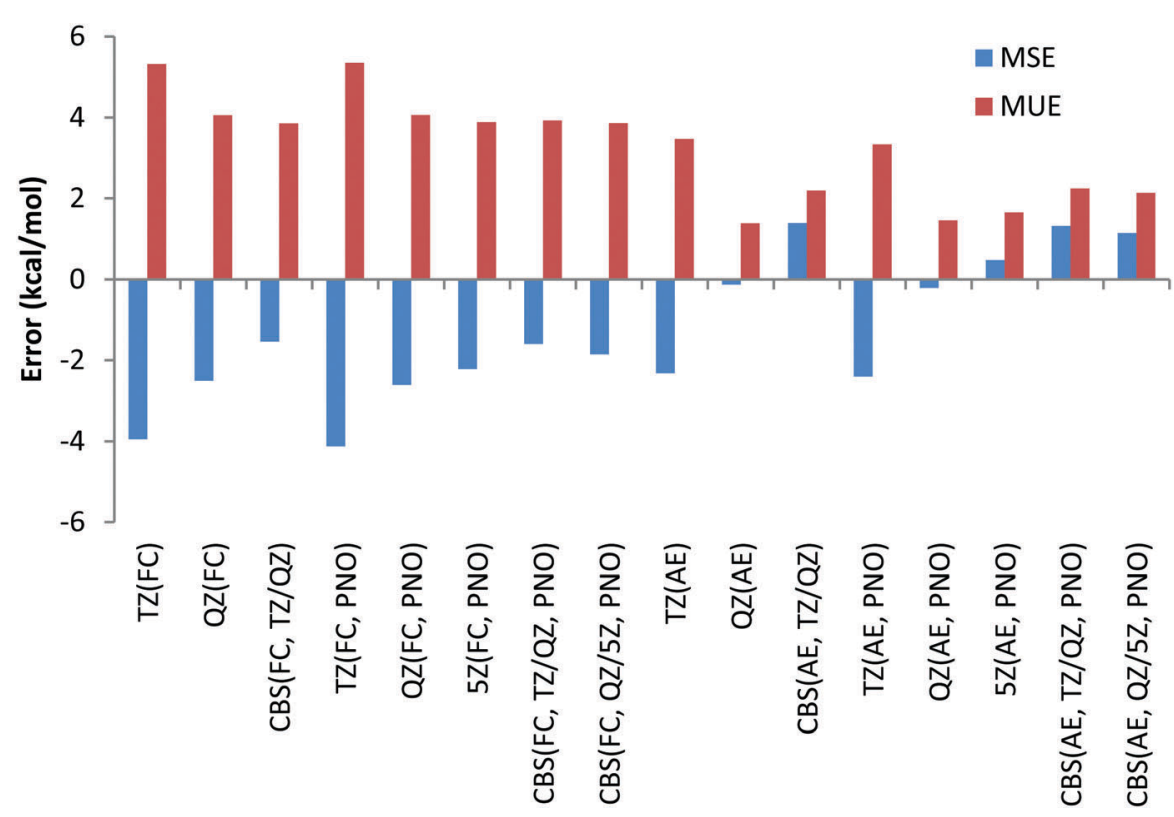

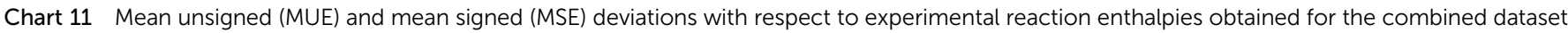

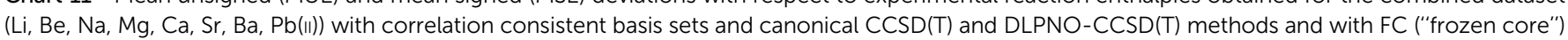
and AE ("all electron") approaches. 
be revisited and the influence on the accuracy of the prediction of the experimental data will be investigated in a different publication. $^{51}$

In the $\mathrm{AE}$ approach the largest errors were obtained with the TZ basis sets, namely 3.5 and $3.3 \mathrm{kcal} \mathrm{mol}^{-1}$ for canonical CCSD(T) and its DLPNO counterpart, respectively. QZ basis sets give MUEs of $1.4 \mathrm{kcal} \mathrm{mol}^{-1}$ for canonical $\operatorname{CCSD}(\mathrm{T})$ and $1.5 \mathrm{kcal} \mathrm{mol}^{-1}$ for DLPNO-CCSD(T) methods. CBS TZ/QZ extrapolation leads to somewhat larger MUE compared to that with the QZ basis set, namely deviations of $2.2 \mathrm{kcal} \mathrm{mol}^{-1}$ for both the canonical and DLPNO coupled cluster, respectively. The $5 \mathrm{Z}$ AE DLPNO-CCSD(T) calculations result in a MUE of $1.7 \mathrm{kcal} \mathrm{mol}^{-1}$ which is $0.2 \mathrm{kcal} \mathrm{mol}^{-1}$ larger compared to QZ results. Finally, the CBS QZ/5Z extrapolation results in a MUE of $2.1 \mathrm{kcal} \mathrm{mol}^{-1}$ which is almost equal to CBS TZ/QZ extrapolation results. The smallest MSEs have been obtained for the QZ basis sets as well when the AE approach is used.

Finally, we have also calculated the deviations between the DLPNO-CCSD $(\mathrm{T})$ reaction energies and their canonical CCSD $(\mathrm{T})$ counterparts with all basis sets and the core-correlation settings for all 32 reactions studied in the present work. The deviations are given in Table S1 (ESI $\dagger$ ) and the summary including the mean unsigned errors (MUE) and mean signed errors (MSE) is depicted in Chart 12. The largest MUE of $0.3 \mathrm{kcal} \mathrm{mol}^{-1}$ has been obtained for CBS extrapolated AE calculations; for all other core correlation settings and basis sets, MUEs of only $0.2 \mathrm{kcal} \mathrm{mol}^{-1}$ have been obtained. When analysing the deviations for the individual reactions in Table $\mathrm{S} 1$ (ESI $\dagger$ ), the largest deviation between DLPNO-CCSD(T) and canonical $\operatorname{CCSD}(\mathrm{T})$ methods has been detected for $\mathrm{AE}$ calculations of reaction energy for reaction (29), see Table 2, and amounts to only $1.1 \mathrm{kcal} \mathrm{mol}^{-1}$. For all

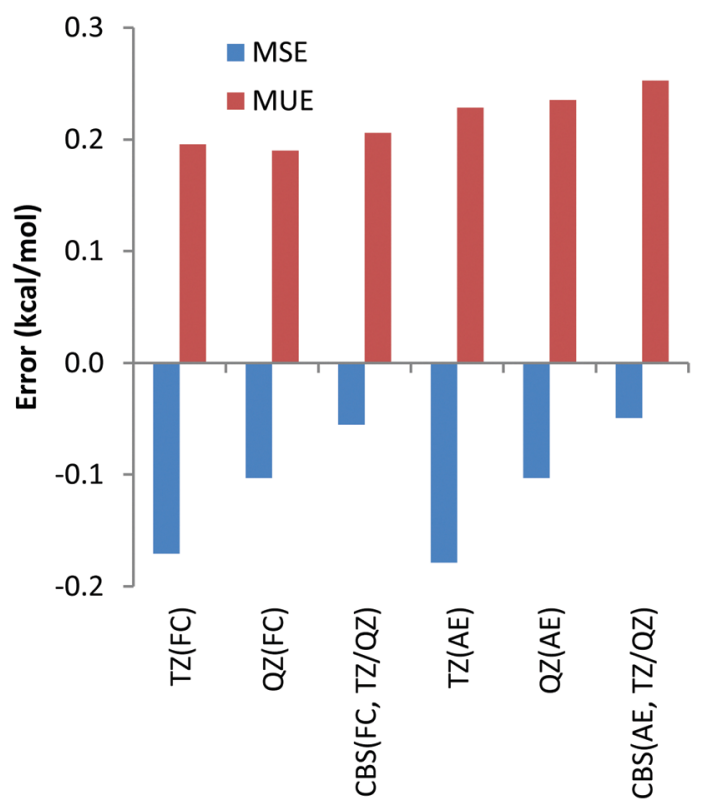

Chart 12 Mean unsigned (MUE) and mean signed (MSE) deviations of the DLPNO-CCSD $(T)$ reaction enthalpies with respect to canonical CCSD(T) counterparts obtained for the combined dataset ( $\mathrm{Li}, \mathrm{Be}, \mathrm{Na}, \mathrm{Mg}, \mathrm{Ca}, \mathrm{Sr}, \mathrm{Ba}$, $\mathrm{Pb}($ II)) with correlation consistent basis sets and with FC ("frozen core") and AE ("all electron") approaches. other reactions for all the combinations of basis sets and core correlation settings, deviations smaller than $0.9 \mathrm{kcal} \mathrm{mol}^{-1}$ have been obtained. All these results indicate that the DLPNO$\operatorname{CCSD}(\mathrm{T})$ method is quite robust and reliable, providing essentially $\operatorname{CCSD}(\mathrm{T})$ accuracy at significantly smaller computational time and less severe hardware demands.

\section{Conclusions}

We have tested the performance of $\operatorname{CCSD}(\mathrm{T})$ and DLPNO$\operatorname{CCSD}(\mathrm{T})$ methods, in conjunction with correlation consistent basis sets of Peterson et al., in reproducing the ligand exchange and association/dissociation enthalpies of highly ionic complexes of $\mathrm{Li}, \mathrm{Be}, \mathrm{Na}, \mathrm{Mg}, \mathrm{Ca}, \mathrm{Sr}, \mathrm{Ba}$ and $\mathrm{Pb}(\mathrm{II})$, for which experimental estimates are available. Two different strategies have been used to determine the number of electrons to be included in the correlation treatment. In the first option, only valence electrons were included, giving rise to the FC approach that can significantly speed up the calculations and either save the CPU time or make the calculations of larger molecules feasible. In the second option, all non-ECP electrons were included, giving rise to the $\mathrm{AE}$ (all electron) approach. The FC approach resulted in poor performance, both due to artificial mixing of the core sub-valence orbitals of the metal atom and the valence orbitals of oxygen and halogens in the molecular core orbitals and due to neglecting core-core and core-valence correlation effects. This is the consequence of very few valence electrons on the metals and highly ionic bonding. However, the performance of the FC approach is not homogeneous and turned out to be surprisingly good with MUEs below $2.0 \mathrm{kcal} \mathrm{mol}^{-1}$ for all the reaction enthalpies of $\mathrm{Li}$ and $\mathrm{Be}$ and for some reaction enthalpies of $\mathrm{Na}, \mathrm{Mg}, \mathrm{Ca}, \mathrm{Sr}, \mathrm{Ba}$ and $\mathrm{Pb}$ complexes.

For a few reaction enthalpies of $\mathrm{Ca}$ and $\mathrm{Ba}$, deviations up to $40 \mathrm{kcal} \mathrm{mol}^{-1}$ have been obtained, indicating that the reliability of the FC approach has always to be verified via test calculations (e.g. on smaller model systems) using $\mathrm{AE}$ calculations or FC calculations with sub-valence orbitals included in the correlation space. Even if no significant artificial mixing is obtained between the core metal orbitals and valence orbitals of oxygen and halogens, the effects of the core-core and corevalence correlations might be as large as $10 \mathrm{kcal} \mathrm{mol}^{-1}$ and cannot be ignored. We realize that, for the considered reactions, the importance of the inclusion of the core orbitals might be obvious and documented in earlier works, and in fact the FC approach resulted in huge deviations from both experimental measurements and $\mathrm{AE}$ calculations. At the same time, we believe that there are many cases where the importance of inclusion of the core correlation and sub-valence orbitals in the calculations is not that obvious and still may result in deviations that are harder to detect. This is especially true for the systems which have never been studied by the $\operatorname{CCSD}(\mathrm{T})$ calculations before, and for which the local $\operatorname{CCSD}(\mathrm{T})$ methods, in particular DLPNO-CCSD(T), made it possible for the first time. The AE approach provided much better overall performance and is recommended for routine $\operatorname{CCSD}(\mathrm{T})$ calculations when 
the importance of the core correlation is unclear, and the right answer for the right reason is the goal. For some of the investigated metals the default chemical cores used in this work might be too large and more conservative chemical core regions might significantly increase the accuracy of results with the FC approach. In a different publication the chemical core regions for heavier elements will be revisited and their influence on the accuracy of the prediction of experimental reaction enthalpies will be investigated. ${ }^{51}$

Another important point is that for most systems results are not converged at the TZ level and require at least QZ basis set calculations. Perhaps surprisingly, both TZ/QZ and QZ/5Z extrapolation schemes, as suggested by Helgaker et al., did not significantly improve upon the QZ results, and in fact often resulted in slightly higher deviations in the reaction enthalpies making CBS extrapolation redundant at least for the reactions in the current work. Moreover, even deviations resulting from $5 \mathrm{Z}$ basis sets turned out to be larger in many cases compared to the QZ results, indicating that some fortuitous cancellations take place.

In general, larger deviations have been obtained for reaction enthalpies involving complexes of heavier metals, i.e. Sr, Ba and $\mathrm{Pb}$. This can at least partially be explained by large experimental uncertainties associated with reaction enthalpies involving these metals.

Finally, the DLPNO-CCSD(T) method with the tight PNO settings was confirmed to very closely approximate the parent canonical $\operatorname{CCSD}(\mathrm{T})$ method performance (MUEs of 0.2 to $0.3 \mathrm{kcal} \mathrm{mol}^{-1}$ ) also for the reaction enthalpies where the effects from the core correlation turned out to be important. This makes the application of the DLPNO method promising also in those cases where core-correlation effects might play an important role. One such potentially promising application is the calculation of unknown formation enthalpies via theoretically calculated reaction enthalpies and known formation enthalpies for all but one reactant and product, as shown by us earlier. ${ }^{88}$ We recommend the use of the DLPNO-CCSD(T) AE protocol for this purpose.

\section{Acknowledgements}

The research reported in this publication was supported by funding from King Abdullah University of Science and Technology (KAUST). For computer time, this research used the resources of the Supercomputing Laboratory at King Abdullah University of Science and Technology (KAUST) in Thuwal, Saudi Arabia. G. Bistoni and A. A. Auer gratefully acknowledge the financial support of the SPP 1807 "Control of London dispersion interactions in molecular chemistry" of the DFG. Open Access funding provided by the Max Planck Society.

\section{Notes and references}

1 K. Raghavachari, G. W. Trucks, J. A. Pople and M. Headgordon, Chem. Phys. Lett., 1989, 157, 479-483.
2 C. J. Cramer, Essentials of Computational Chemistry: Theories and Models, Wiley, 2nd edn, 2005.

3 F. Jensen, Introduction to Computational Chemistry, Wiley, 2nd edn, 2006.

4 C. Hättig and F. Weigend, J. Chem. Phys., 2000, 113, 5154-5161.

5 F. Neese, F. Wennmohs and A. Hansen, J. Chem. Phys., 2009, 130, 114108.

6 H.-J. Werner and M. Schütz, J. Chem. Phys., 2011, 135, 144116.

7 D. G. Liakos, A. Hansen and F. Neese, J. Chem. Theory Comput., 2011, 7, 76-87.

8 J. W. Boughton and P. Pulay, J. Comput. Chem., 1993, 14, 736-740.

9 C. Riplinger and F. Neese, J. Chem. Phys., 2013, 138, 034106.

10 C. Riplinger, B. Sandhoefer, A. Hansen and F. Neese, J. Chem. Phys., 2013, 139, 134101.

11 P. Y. Ayala and G. E. Scuseria, J. Chem. Phys., 1999, 110, 3660-3671.

12 S. Saebo and P. Pulay, Annu. Rev. Phys. Chem., 1993, 44, 213-236.

13 C. Hampel and H. J. Werner, J. Chem. Phys., 1996, 104, 6286-6297.

14 M. Schütz and H. J. Werner, Chem. Phys. Lett., 2000, 318, 370-378.

15 D. G. Liakos, M. Sparta, M. K. Kesharwani, J. M. L. Martin and F. Neese, J. Chem. Theory Comput., 2015, 11, 1525-1539.

16 C. Riplinger, P. Pinski, U. Becker, E. F. Valeev and F. Neese, J. Chem. Phys., 2016, 144, 024109.

17 F. Neese, WIREs Comput. Mol. Sci., 2012, 2, 73-78.

18 Y. Minenkov, E. Chermak and L. Cavallo, J. Chem. Theory Comput., 2015, 11, 4664-4676.

19 M. Sparta, C. Riplinger and F. Neese, J. Chem. Theory Comput., 2014, 10, 1099-1108.

20 M. Sparta and F. Neese, Chem. Soc. Rev., 2014, 43, 5032-5041.

21 C. W. Bauschlicher, C. F. Melius and M. D. Allendorf, J. Chem. Phys., 1999, 110, 1879-1881.

22 A. Schulz, B. J. Smith and L. Radom, J. Phys. Chem. A, 1999, 103, 7522-7527.

23 M. B. Sullivan, M. A. Iron, P. C. Redfern, J. M. L. Martin, L. A. Curtiss and L. Radom, J. Phys. Chem. A, 2003, 107, 5617-5630.

24 D. S. Ranasinghe, M. J. Frisch and G. A. Petersson, J. Chem. Phys., 2015, 143, 214110.

25 A. J. Austin, M. J. Frisch, J. A. Montgomery and G. A. Petersson, Theor. Chem. Acc., 2002, 107, 180-186.

26 R. J. Bartlett and M. Musial, Rev. Mod. Phys., 2007, 79, 291-352.

27 D. Feller, K. A. Peterson and D. A. Dixon, Mol. Phys., 2012, 110, 2381-2399.

28 L. A. Curtiss, K. Raghavachari, G. W. Trucks and J. A. Pople, J. Chem. Phys., 1991, 94, 7221-7230.

29 L. A. Curtiss, P. C. Redfern and K. Raghavachari, WIREs Comput. Mol. Sci., 2011, 1, 810-825.

30 K. A. Peterson and T. H. Dunning, J. Chem. Phys., 2002, 117, 10548-10560.

31 B. P. Prascher, D. E. Woon, K. A. Peterson, T. H. Dunning Jr. and A. K. Wilson, Theor. Chem. Acc., 2011, 128, 69-82.

32 M. A. Iron, M. Oren and J. M. L. Martin, Mol. Phys., 2003, 101, 1345-1361. 
33 Y. Gong, L. Andrews, C. W. Bauschlicher, K. S. Thanthiriwatte and D. A. Dixon, Dalton Trans., 2012, 41, 11706-11715.

34 D. Figgen, K. A. Peterson, M. Dolg and H. Stoll, J. Chem. Phys., 2009, 130, 164108.

35 D. Feller, K. A. Peterson and D. A. Dixon, J. Chem. Phys., 2008, 129, 204105.

36 K. A. Peterson, D. Feller and D. A. Dixon, Theor. Chem. Acc., 2012, 131, 1-20.

37 D. A. Dixon, D. Feller and K. A. Peterson, in Annu. Rep. Comput. Chem., ed. A. W. Ralph, Elsevier, 2012, vol. 8, pp. 1-28.

38 D. Feller, K. A. Peterson and D. A. Dixon, The Impact of Larger Basis Sets and Explicitly Correlated Coupled Cluster Theory on the Feller-Peterson-Dixon Composite Method. In Annual Reports in Computational Chemistry, Elsevier Science, 2016, vol. 12.

39 N. J. DeYonker, T. R. Cundari and A. K. Wilson, J. Chem. Phys., 2006, 124, 104111.

40 A. Karton, E. Rabinovich, J. M. L. Martin and B. Ruscic, J. Chem. Phys., 2006, 125, 144108.

41 D. N. Laikov and Y. A. Ustynyuk, Russ. Chem. Bull., 2005, 54, 820-826.

42 M. J. Frisch, G. W. Trucks, H. B. Schlegel, G. E. Scuseria, M. A. Robb, J. R. Cheeseman, G. Scalmani, V. Barone, B. Mennucci, G. A. Petersson, H. Nakatsuji, M. Caricato, X. Li, H. P. Hratchian, A. F. Izmaylov, J. Bloino, G. Zheng, J. L. Sonnenberg, M. Hada, M. Ehara, K. Toyota, R. Fukuda, J. Hasegawa, M. Ishida, T. Nakajima, Y. Honda, O. Kitao, H. Nakai, T. Vreven, J. A. Montgomery Jr, J. E. Peralta, F. Ogliaro, M. J. Bearpark, J. Heyd, E. N. Brothers, K. N. Kudin, V. N. Staroverov, R. Kobayashi, J. Normand, K. Raghavachari, A. P. Rendell, J. C. Burant, S. S. Iyengar, J. Tomasi, M. Cossi, N. Rega, N. J. Millam, M. Klene, J. E. Knox, J. B. Cross, V. Bakken, C. Adamo, J. Jaramillo, R. Gomperts, R. E. Stratmann, O. Yazyev, A. J. Austin, R. Cammi, C. Pomelli, J. W. Ochterski, R. L. Martin, K. Morokuma, V. G. Zakrzewski, G. A. Voth, P. Salvador, J. J. Dannenberg, S. Dapprich, A. D. Daniels, Ö. Farkas, J. B. Foresman, J. V. Ortiz, J. Cioslowski and D. J. Fox, Gaussian 09, Revision D.01, Gaussian, Inc., Wallingford CT, 2013.

43 X. Xu, W. Zhang, M. Tang and D. G. Truhlar, J. Chem. Theory Comput., 2015, 11, 2036-2052.

44 L. Cheng, J. Gauss, B. Ruscic, P. B. Armentrout and J. F. Stanton, J. Chem. Theory Comput., 2017, 13, 1044-1056.

45 Z. Fang, M. Vasiliu, K. A. Peterson and D. A. Dixon, J. Chem. Theory Comput., 2017, 13, 1057-1068.

46 Y. Minenkov, E. Chermak and L. Cavallo, J. Chem. Theory Comput., 2016, 12, 1542-1560.

47 J. P. Perdew, K. Burke and M. Ernzerhof, Phys. Rev. Lett., 1996, 77, 3865-3868.

48 J. P. Perdew, K. Burke and M. Ernzerhof, Phys. Rev. Lett., 1997, 78, 1396.

49 D. N. Laikov, Chem. Phys. Lett., 2005, 416, 116-120.

50 K. G. Dyall, J. Chem. Phys., 1994, 100, 2118-2127.

51 Manuscript in preparation.

52 T. H. Dunning, J. Chem. Phys., 1989, 90, 1007-1023.
53 J. Koput and K. A. Peterson, J. Phys. Chem. A, 2002, 106, 9595-9599.

54 H. Li, H. Feng, W. Sun, Y. Zhang, Q. Fan, K. A. Peterson, Y. Xie and H. F. Schaefer III, Mol. Phys., 2013, 111, 2292-2298.

55 I. S. Lim, H. Stoll and P. Schwerdtfeger, J. Chem. Phys., 2006, 124, 034107.

56 K. A. Peterson and K. E. Yousaf, J. Chem. Phys., 2010, 133, 174116.

57 K. A. Peterson, D. Figgen, E. Goll, H. Stoll and M. Dolg, J. Chem. Phys., 2003, 119, 11113-11123.

58 K. A. Peterson, B. C. Shepler, D. Figgen and H. Stoll, J. Phys. Chem. A, 2006, 110, 13877-13883.

59 B. Metz, H. Stoll and M. Dolg, J. Chem. Phys., 2000, 113, 2563-2569.

60 C. Hättig, Phys. Chem. Chem. Phys., 2005, 7, 59-66.

61 A. Hellweg, C. Hättig, S. Hoefener and W. Klopper, Theor. Chem. Acc., 2007, 117, 587-597.

62 COSMOLOGIC, http://cosmologic-services.de/basis-sets/basis sets.php.

63 G. L. Stoychev, A. A. Auer and F. Neese, J. Chem. Theory Comput., 2017, 13, 554-562.

64 A. Halkier, T. Helgaker, P. Jørgensen, W. Klopper, H. Koch, J. Olsen and A. K. Wilson, Chem. Phys. Lett., 1998, 286, 243-252.

65 T. Helgaker, W. Klopper, H. Koch and J. Noga, J. Chem. Phys., 1997, 106, 9639-9646.

66 A. Halkier, T. Helgaker, P. Jørgensen, W. Klopper and J. Olsen, Chem. Phys. Lett., 1999, 302, 437-446.

67 NIST Chemistry WebBook, NIST Standard Reference Database Number 69, National Institute of Standards and Technology, 2005.

68 M. W. Chase Jr, J. Phys. Chem. Ref. Data, Monogr., 1998, 9, 1-1951.

69 M. N. Glukhovtsev, R. D. Bach and C. J. Nagel, J. Phys. Chem. A, 1997, 101, 316-323.

70 V. L. Chevrier, S. P. Ong, R. Armiento, M. K. Y. Chan and G. Ceder, Phys. Rev. B: Condens. Matter Mater. Phys., 2010, 82, 075122.

71 K. E. Riley and K. M. Merz, J. Phys. Chem. A, 2007, 111, 6044-6053.

72 R. H. West, G. J. O. Beran, W. H. Green and M. Kraft, J. Phys. Chem. A, 2007, 111, 3560-3565.

73 W. J. Zhang, D. G. Truhlar and M. S. Tang, J. Chem. Theory Comput., 2013, 9, 3965-3977.

74 R. D. Bach, D. S. Shobe, H. B. Schlegel and C. J. Nagel, J. Phys. Chem., 1996, 100, 8770-8776.

75 N. J. DeYonker, T. G. Williams, A. E. Imel, T. R. Cundari and A. K. Wilson, J. Chem. Phys., 2009, 131, 024106.

76 K. S. Krasnov, Molecular Constants of Inorganic Compounds, Reference Book, Khimia, Leningrad, 1979.

77 L. V. Gurvich, G. V. Karachevtsev, V. N. Kondrat'ev, J. A. Lebedev, V. A. Medvedev, V. K. Potapov and Y. S. Khodeev, Bond dissociation enrgies, ionization potentials and electron affinities, Nauka, Moscow, 1974.

78 V. P. Glushko, Thermodynamic properties of individual substances, Nauka, Moscow, 1979.

79 O. M. Nefedov, M. P. Egorov, A. I. Ioffe, L. G. Menchikov, P. S. Zuev, V. I. Minkin, B. Y. Simkin and M. N. Glukhovtsev, Pure Appl. Chem., 1992, 64, 265-314. 
80 K. S. Thanthiriwatte, M. Vasiliu, S. R. Battey, Q. Lu, K. A. Peterson, L. Andrews and D. A. Dixon, J. Phys. Chem. A, 2015, 119, 5790-5803.

81 V. P. Glushko, et al., Thermodynamic properties of individual substances, NAUKA, Moscow, 1982.

82 M. Vasiliu, S. G. Li, K. A. Peterson, D. Feller, J. L. Gole and D. A. Dixon, J. Phys. Chem. A, 2010, 114, 4272-4281.

83 V. P. Glushko, Thermodynamic properties of individual substances, NAUKA, Moscow, 1981.
84 M. Vasiliu, D. Feller, J. L. Gole and D. A. Dixon, J. Phys. Chem. A, 2010, 114, 9349-9358.

85 F. Weigend and R. Ahlrichs, Phys. Chem. Chem. Phys., 2005, 7, 3297-3305.

86 T. Noro, M. Sekiya and T. Koga, Theor. Chem. Acc., 2012, 131, 1124.

87 F. Neese, ORCA 3.0 An ab initio, DFT and semiempirical SCFMO package. Input description., 2013.

88 Y. Minenkov, V. V. Sliznev and L. Cavallo, Inorg. Chem., 2017, 56, 1386-1401. 